\title{
The Cauchy Problem for Coupled Yang-Mills and Scalar Fields in the Temporal Gauge
}

\author{
J. Ginibre ${ }^{1}$ and G. Velo ${ }^{2}$ \\ 1 Laboratoire de Physique Théorique et Hautes Energies*, Université de Paris-Sud, F-91405 Orsay, \\ France \\ 2 Istituto di Fisica A. Righi, Università di Bologna and I. N. F. N., Sezione di Bologna, \\ I-40126 Bologna, Italy
}

\begin{abstract}
We study the Cauchy problem for minimally coupled classical Yang-Mills and scalar fields in $n+1$ dimensional space-time in the temporal gauge. We prove the existence and uniqueness of solutions for small time intervals and for any $n$. We then develop a general theory of solutions in local spaces and extend the previous local (in time) results to this more general setting. In space-time dimensions two and three, we prove the existence of global (in time) solutions by the method of a priori estimates, both in global and local spaces. In space-time dimension four, our estimates yield only partial results on the global existence problem.
\end{abstract}

\section{Introduction}

In spite of the large amount of work which has been devoted in the last years to the Yang-Mills equations, a satisfactory understanding of the properties of their solutions is still lacking. At the classical level most of the effort has gone into the Euclidean version of the theory, partly because of the richness of its geometrical structure, and partly because of its possible relevance to the quantized theory. On the other hand the theory in Minkowski space-time poses an interesting and non-trivial problem from the point of view of non-linear partial differential equations, and may appear as the classical limit of the quantized theory. The equations in Minkowski space-time have been studied most notably by Segal [11] who has considered the Cauchy problem for the pure Yang-Mills theory in four dimensional space-time, in the so-called temporal gauge $A^{0}=0$, giving a proof of the existence and uniqueness of solutions of the Cauchy problem for small time intervals. More recently, for coupled Yang-Mills and scalar fields in four dimensions, Glassey and Strauss have studied the time decay of solutions [4], [5] and have obtained a class of global solutions of a special type suggested by the known

* Laboratoire associé au Centre National de la Recherche Scientifique 
explicit static solutions [6]. A global existence proof for the Cauchy problem with general initial data in four dimensional space-time would be of great interest. ${ }^{1}$

In this and in a subsequent paper we take up the Cauchy problem for minimally coupled Yang-Mills and scalar fields in $n+1$ dimensional space-time and extend the previous results in two directions. One concerns the local problem and is inspired by two remarks. First the Yang-Mills equations describe propagation at finite speed; it is therefore unnatural to impose restrictions on the behaviour at infinity of the initial data and of the solutions. A more natural setting consists of working in local spaces as was done in [9]. The local theory developed in [9] however was restricted to uniformly locally bounded initial data and solutions. In the present paper we extend the local theory to remove this restriction and apply it to the equations under consideration. This extended local theory also provides a convenient starting point to study the "boost" problem considered in a more general setting by Christodoulou [2]. The second remark that leads us to develop a theory in local spaces is that the Yang-Mills equations carry with them a nontrivial elliptic constraint which may, and in general will, produce long range effects, especially in low dimensions. These long range effects may impose severe and unnecessary restrictions on the initial data and on the solutions if one insists on working in global spaces.

The second direction of our investigation is concerned with the global (in time) existence problem. In this paper we prove the existence of global solutions for coupled Yang-Mills and scalar fields in the temporal gauge in two and three spacetime dimensions, and obtain some partial results in space-time dimension four. In the following paper we prove the existence of global solutions in two dimensional space-time both for the coupled (possibly massive) Yang-Mills and scalar fields in the Lorentz gauge and for a number of related models, namely for the so-called $O(N), \mathbb{C} P(N)$, and $G \mathbb{C}(N, p)$ models. All these global (in time) existence results hold both for the theory in global and in local spaces. The proof of the existence of global results uses the standard method of a priori estimates in the form recently developed by Moncrief [7] to treat the case of scalar electrodynamics in three space-time dimensions. An important role in performing the estimates is played by the Sobolev inequalities with covariant derivatives.

The paper is organized as follows. In Sect. 2 we introduce the basic notation, write the equations, choose the dynamical variables and treat the local problem in global spaces for arbitrary space-time dimension. In particular we prove the existence, uniqueness and regularity of the solutions. This section is a straightforward extension of Segal's work [10], [11]. In Sect. 3 we develop at a semiabstract level a general theory of local (in time) existence and uniqueness in local spaces. In Sect. 4 we apply this theory to the equations for coupled Yang-Mills and scalar fields in the temporal gauge, for arbitrary space-time dimension. In Sect. 5 we study the problem of global existence: we derive the relevant estimates and use them to prove the existence of global solutions both in global and local spaces for space-time dimensions two and three. For space time dimension four our

1 After this paper was completed, we were informed by V. Moncrief that he and D. Eardley have succeeded in obtaining such a proof [12] 
estimates prove that the solutions are either global or fairly irregular. The covariant Sobolev inequalities are proved in the Appendix.

The results of this paper and part of those of the subsequent paper were announced in [3].

\section{The Cauchy Problem in Global Spaces for Small Time Intervals}

In this section we begin our study of the initial value problem for the classical Yang-Mills field minimally coupled to a scalar field, in the temporal gauge $A_{0}=0$. We first introduce some notation. The Yang-Mills potential $A_{\mu}(t, x)$ is a function from $n+1$ dimensional space time to the Lie algebra $\mathscr{G}$ of a compact Lie group $G$. The corresponding field is $F_{\mu \nu}=\partial_{\mu} A_{v}-\partial_{\nu} A_{\mu}+e\left[A_{\mu}, A_{\nu}\right]$, where [,] denotes the commutator in $\mathscr{G}$. We assume the existence of a non-degenerate positive definite bilinear form in $\mathscr{G}$, denoted by $\langle.,$.$\rangle , invariant under the ad-$ joint representation of the group. If $G$ is semisimple one can take the Killing form; if $G$ is commutative one can take any positive definite form on $\mathscr{G}$. The scalar field $\phi(t, x)$ belongs to a unitary representation of $G$ in a finite dimensional complex vector space $\mathscr{F}$. We also denote by $\langle.,$.$\rangle the invariant scalar product in \mathscr{F}$ and we use the same notation for an element of $\mathscr{G}$ and for its representative in $\mathscr{F}$. We shall write $\langle B, B\rangle=|B|^{2}$. We use the same notation $D_{\mu}$ for the covariant derivative in $\mathscr{G}$, where $D_{\mu}=\partial_{\mu}+e\left[A_{\mu}, \cdot\right]$ and in $\mathscr{F}$, where $D_{\mu}^{\mu}=\partial_{\mu}+e A_{\mu}$. We use the metric $g_{\mu \nu}$ with $g_{00}=1, g_{i i}=-1, g_{\mu \nu}=0$ for $v \neq \mu$.

The field equations are the variational equations associated with the Lagrangian density

$$
\mathscr{L}=-\frac{1}{4}\left\langle F_{\mu \nu}, F^{\mu v}\right\rangle+\left\langle D_{\mu} \phi, D^{\mu} \phi\right\rangle-V\left(|\phi|^{2}\right),
$$

where $V$ is a real $\mathscr{C}^{1}$ function defined in $\mathbb{R}^{+}$with $V(0)=0$. The equations are

$$
\begin{gathered}
K^{\mu} \equiv D_{\lambda} F^{\lambda \mu}+J^{\mu}=0, \\
M \equiv D_{\mu} D^{\mu} \phi+\phi V^{\prime}\left(|\phi|^{2}\right)=0,
\end{gathered}
$$

where $J^{\mu} \in \mathscr{G}$ is defined by $\left\langle J^{\mu}, C\right\rangle=2 e \operatorname{Re}\left\langle D^{\mu} \phi, C \phi\right\rangle$ for all $C \in \mathscr{G}$, and $V^{\prime}$ is the derivative of $V$. As is well known, in order to convert these equations into an evolution equation for a suitable set of dynamical variables, ones needs to add a gauge condition on the potentials. Here we choose the temporal gauge $A_{0}=0$. It can be shown that any suitably regular potential $A_{\mu}$ can be transformed into a new potential with $A_{0}=0$ by a gauge transformation [11]. In this gauge we choose as dynamical variables the quantities $A=\left\{A_{j}, 1 \leqq j \leqq n\right\}, F_{0}=\left\{F_{0 j}, 1 \leqq j \leqq n\right\}$, $F=\left\{F_{j k}, 1 \leqq j<k \leqq n\right\}, \phi, \psi_{0}$ and $\psi=\left\{\psi_{j}, 1 \leqq j \leqq n\right\}\left(\psi_{\mu}\right.$ has to be thought of as $D_{\mu} \phi$ ). The variational equations can be conveniently rewritten as the following first-order system of equations of motion

$$
\partial_{0}\left(\begin{array}{c}
A \\
F_{0} \\
F
\end{array}\right)=\left(\begin{array}{ccc}
0 & \mathbb{1} & 0 \\
0 & 0 & R_{A} \\
0 & -R_{A}^{*} & 0
\end{array}\right)\left(\begin{array}{c}
A \\
F_{0} \\
F
\end{array}\right)+\left(\begin{array}{c}
0 \\
-e\left[A^{k}, F_{k} .\right]-J \\
e\left[A, F_{0}\right]+e\left[F_{0}, A\right]
\end{array}\right),
$$




$$
\partial_{0}\left(\begin{array}{l}
\phi \\
\psi_{0} \\
\psi
\end{array}\right)=\left(\begin{array}{ccc}
0 & 1 & 0 \\
0 & 0 & R \\
0 & -R^{*} & 0
\end{array}\right)\left(\begin{array}{l}
\phi \\
\psi_{0} \\
\psi
\end{array}\right)+\left(\begin{array}{c}
0 \\
-e A^{k} \psi_{k}-\phi V^{\prime} \\
e\left(A \psi_{0}+F_{0} \phi\right)
\end{array}\right)
$$

supplemented by the constraints

$$
\begin{aligned}
F_{j k} & =\partial_{j} A_{k}-\partial_{k} A_{j}+e\left[A_{j}, A_{k}\right], \\
K_{0} & =0 \\
\psi_{j} & =D_{j} \phi .
\end{aligned}
$$

Here $J_{0}$ and $J=\left\{J_{j}, 1 \leqq j \leqq n\right\}$ are supposed to be expressed in terms of $\phi, \psi_{0}$ and $\psi_{j} ; R_{A}$ is the $n \times n(n-1) / 2$ matrix operator with entries $\left(R_{A}\right)_{j}^{k \ell}=-\delta_{j}^{\ell} \partial^{k}+$ $\delta_{j}^{k} \partial^{\ell}$ and $R$ is the $1 \times n$ matrix operator with entries $(R)_{0}^{j}=-\partial^{j}$. The system of equations (2.4) and (2.5) can be written more compactly as

$$
\partial_{0} u(t)=T u(t)+f(u(t))
$$

where

$$
\begin{gathered}
u=\left(\begin{array}{l}
u_{A} \\
u_{\phi}
\end{array}\right), u_{A}=\left(\begin{array}{c}
A \\
F_{0} \\
F
\end{array}\right), u_{\phi}=\left(\begin{array}{l}
\phi \\
\psi_{0} \\
\psi
\end{array}\right), \\
T=\left(\begin{array}{cc}
T_{A} & 0 \\
0 & T_{\phi}
\end{array}\right), f=\left(\begin{array}{l}
f_{A} \\
f_{\phi}
\end{array}\right),
\end{gathered}
$$

and $T_{A}, T_{\phi}, f_{A}$,

and $f_{\phi}$ can be read directly from (2.4) and (2.5). The fields $u_{A}$ and $u_{\phi}$ take values in the finite dimensional vector spaces $\mathscr{V}_{A}=\mathscr{G} \otimes \mathbb{R}^{2 n+n(n-1) / 2}$ and $\mathscr{V}_{\phi}=\mathscr{F} \otimes \mathbb{C}^{2+n}$ respectively, so that $u$ takes values in $\mathscr{V}=\mathscr{V}_{A} \oplus \mathscr{V}_{\phi}$. The Cauchy problem for the equation (2.9) can be transformed into the following integral equation

$$
u(t)=U(t) u_{0}+\int_{0}^{t} d \tau U(t-\tau) f(u(\tau))
$$

where $u_{0}$ is the initial condition,

$$
\begin{gathered}
U(t)=\left(\begin{array}{ll}
U_{A}(t) & 0 \\
0 & U_{\phi}(t)
\end{array}\right), \\
U_{A}(t)=\left(\begin{array}{lll}
1 & \omega_{\perp}^{-1} \sin \omega_{\perp} t & \left(1-\cos \omega_{\perp} t\right) \omega_{\perp}^{-2} R_{A} \\
0 & \cos \omega_{\perp} t & \left(\sin \omega_{\perp} t\right) \omega_{\perp}^{-1} R_{A} \\
0-R_{A}^{*} \omega_{\perp}^{-1} \sin \omega_{\perp} t & \cos \left(R_{A}^{*} R_{A}\right)^{1 / 2} t
\end{array}\right)
\end{gathered}
$$

with $\omega_{\perp}=\left(R_{A} R_{A}^{*}\right)^{1 / 2}$ or equivalently $\left(\omega_{\perp}^{2}\right)_{i}^{j}=-\partial_{i} \partial^{j}-\Delta \delta_{i}^{j}$, and

$$
U_{\phi}(t)=\left(\begin{array}{lll}
1 & \omega^{-1} \sin \omega t & (1-\cos \omega t) \omega^{-2} R \\
0 & \cos \omega t & (\sin \omega t) \omega^{-1} R \\
0-R^{*} \omega^{-1} \sin \omega t & \cos \left(R^{*} R\right)^{1 / 2} t
\end{array}\right)
$$

with $\omega=\left(R R^{*}\right)^{1 / 2}=(-\Delta)^{1 / 2}$.

In this section we look for solutions $u(t)$ of (2.11) as continuous functions of $t$ with values in the space $\mathscr{H}^{k} \equiv \mathscr{H}^{k}\left(\mathbb{R}^{n}\right)$ (where $k$ is a non-negative integer) of 
functions from $\mathbb{R}^{n}$ to $\mathscr{V}$ for which

$$
\|u\|^{2}=\sum_{\sigma} \sum_{|\alpha| \leqq k}\left\|\left|\partial^{\alpha} u_{\sigma}\right|\right\|_{2}^{2}<\infty .
$$

Here $\alpha$ is a multiindex and $\sigma$ labels the components of $u$ corresponding to the decomposition (2.10) and, possibly, for each term in this decomposition, to its various components in an orthonormal basis of $\mathbb{R}^{n}$, so that, for each $\sigma, u_{\sigma}$ takes its values in $\mathscr{G}$ or $\mathscr{F}$. Finally $\|\cdot\|_{q}$ denotes the norm in $L^{q}\left(\mathbb{R}^{n}\right)$. The space $\mathscr{H}^{k}\left(\mathbb{R}^{n}\right)$ is a direct sum of usual Sobolev spaces.

In order to prove the existence of solutions of (2.11) we need the following properties of $U(t)$.

Lemma 2.1. For any $k, U(t)$ is a (bounded) strongly continuous one parameter group in $\mathscr{H}^{k}$ and, for any $t \in \mathbb{R}$, for any $u \in \mathscr{H}^{k}, U(t)$ satisfies the estimate

where

$$
\|U(t) u\| \leqq \mu(t)\|u\|
$$

$$
\mu(t)=\left\{1+\frac{1}{2}|t|\left(|t|+\left(t^{2}+4\right)^{1 / 2}\right)\right\}^{1 / 2} .
$$

Proof. It will be sufficient to prove (2.14) for smooth $u$. The estimate is satisfied separately by $U_{A}(t)$ and $U_{\phi}(t)$. We prove it only for $U_{A}(t)$, the proof for $U_{\phi}(t)$ being similar. Since $U_{A}(t)$ essentially describes the evolution under the free Maxwell equations, it is well known that, for any multiindex $\alpha$ of space derivatives, the quantity

$$
M(t)^{2}=\sum_{1 \leqq j \leqq n}\left\|\left|\partial^{\alpha} F_{0 j}(t)\right|\right\|_{2}^{2}+\sum_{j<k}\left\|\left|\partial^{\alpha} F_{j k}(t)\right|\right\|_{2}^{2}
$$

is time independent under the evolution described by $U_{A}(t)$. We next consider the quantity

which satisfies

$$
N(t)^{2}=\sum_{1 \leqq j \leqq n}\left\|\left|\partial^{\alpha} A_{j}(t)\right|\right\|_{2}^{2},
$$

Therefore

$$
\left|\partial_{0} N(t)^{2}\right|=2\left|\int d x\left\langle\partial^{\alpha} A^{j}, \partial^{\alpha} F_{0 j}\right\rangle\right|
$$

$$
\leqq 2 M(t) N(t)
$$

so that

$$
N(t) \leqq N(0)+M(0)|t|,
$$

$$
\begin{aligned}
M(t)^{2}+N(t)^{2} & \leqq M(0)^{2}+(N(0)+M(0)|t|)^{2} \\
& \leqq\left(M(0)^{2}+N(0)^{2}\right) \mu(t)^{2}
\end{aligned}
$$

where

$$
\mu(t)^{2}=\operatorname{Sup}_{a>0}\left\{1+(a+|t|)^{2}\right\}\left(1+a^{2}\right)^{-1} .
$$

By an elementary computation (2.21) yields (2.15). The required estimate for $U_{A}(t)$ follows from $(2.16),(2.17)$ and $(2.20)$.

We can now prove the existence of local solutions of (2.11). For any interval 
$I$ and any Banach space $\mathscr{B}$ we denote by $\mathscr{C}(I, \mathscr{B})$ the space of continuous functions from $I$ to $\mathscr{B}$, and, for any positive integer $\ell$, we denote by $\mathscr{C}^{\ell}(I, \mathscr{B})$ the space of $\ell$-times continuously differentiable functions from $I$ to $\mathscr{B}$. For compact $I, \mathscr{C}(I, \mathscr{B})$ is a Banach space when equipped with the Sup norm.

Proposition 2.1. Let $k=[n / 2+1]([\lambda]$ is the integral part of $\lambda), V \in \mathscr{C}^{k+2}\left(\mathbb{R}^{+}\right)$ and $u_{0} \in \mathscr{H}^{k}$. Then there exists $T_{0}>0$, depending on $\left\|u_{0}\right\|$, such that (2.11) has a unique solution $u \in \mathscr{C}\left(\left[-T_{0}, T_{0}\right], \mathscr{H}^{k}\right)$.

Proof. The result is a straightforward application of the methods of [10], using Lemma 2.1 and the fact that $f$ satisfies a Lipschitz condition, namely

$$
\|f(u)-f(v)\| \leqq C_{0}(\rho)\|u-v\|
$$

for all $u, v \in \mathscr{H}^{k}$ such that $\|u\|,\|v\| \leqq \rho$, where $C_{0}(\cdot)$ is an increasing real function. This in turn follows from the assumption $V \in \mathscr{C}^{k+2}\left(\mathbb{R}^{+}\right)$, from the fact that the terms in $f$ not containing $V$ are bilinear in the components of $u$, and from the fact that the Sobolev space $H^{k}\left(\mathbb{R}^{n}\right)$ is a Banach algebra for $k>n / 2([1]$ Theorem 5.23 p. 115).

Q.E.D.

If the initial data and the interaction potential $V$ satisfy additional regularity properties, the solution is also more regular.

Proposition 2.2. Let $k^{\prime} \geqq k=[n / 2+1]$, let $V \in \mathscr{C}^{k^{\prime}+1}\left(\mathbb{R}^{+}\right)$and let $u_{0} \in \mathscr{H}^{k^{\prime}}$. Let I be an interval of $\mathbb{R}$ containing the origin and let $u \in \mathscr{C}\left(I, \mathscr{H}^{k}\right)$ be a solution of (2.11). Then $u \in \mathscr{C}^{\ell}\left(I, \mathscr{H}^{k^{\prime}-\ell}\right)$ for any $\ell, 0 \leqq \ell \leqq k^{\prime}$.

Proof. First we prove that $u \in \mathscr{C}\left(I, \mathscr{H}^{k^{\prime}}\right)$ by induction. Suppose that $u \in \mathscr{C}\left(I, H^{\ell-1}\right)$ for some $\ell, k<\ell \leqq k^{\prime}$. We want to show that $u \in \mathscr{C}\left(I, \mathscr{H}^{\ell}\right)$ or equivalently that, for any multiindex $\alpha$ with $|\alpha|=\ell-k, \partial^{\alpha} u \in \mathscr{C}\left(I, \mathscr{H}^{k}\right)$. Let $v^{\alpha}=\partial^{\alpha} u$. Then $v^{\alpha} \in \mathscr{C}\left(I, \mathscr{H}^{k-1}\right)$ and $v^{\alpha}$ satisfies the equation

$$
v^{\alpha}(t)=U(t) \partial^{\alpha} u_{0}+\int_{0}^{t} d \tau U(t-\tau)\left\{\sum_{\sigma} \frac{\partial b}{\partial u_{\sigma}}(u(\tau)) v_{\sigma}^{\alpha}(\tau)+g^{\alpha}(u(\tau))\right\}
$$

where $g^{\alpha}$ is a polynomial in the space derivatives of $u$ of order at most $\ell-k-1$ and in the derivatives of $f$ with respect to $u$ of order at most $\ell-k$. From the assumption on $V$, the induction assumption, and the fact that $H^{k}\left(\mathbb{R}^{n}\right)$ is an algebra, it follows that $g^{\alpha}(u(\tau))$ and $\frac{\partial f}{\partial u_{\sigma}}(u(\tau))$ belong to $\mathscr{C}\left(I, \mathscr{H}^{k}\right)$. Furthermore the inhomogeneous term $U(t) \partial^{\alpha} u_{0}$ belongs to $\mathscr{C}\left(I, \mathscr{H}^{k}\right)$ by assumption. Therefore $(2.23)$ considered as a linear integral equation for $v^{\alpha}$ has a unique solution both in $\mathscr{C}\left(I, \mathscr{H}^{k}\right)$ and in $\mathscr{C}\left(I, \mathscr{H}^{k-1}\right)$. These two solutions coincide and therefore $\partial^{\alpha} u \in$ $\mathscr{C}\left(I, \mathscr{H}^{k}\right)$. This completes the induction proof that $u \in \mathscr{C}\left(I, \mathscr{H}^{k^{\prime}}\right)$.

We can now prove Proposition 2.2 by induction on $\ell$. Suppose $u \in \bigcap_{0 \leqq j \leqq \ell-1}$ $\mathscr{C}^{j}\left(I, \mathscr{H}^{k^{\prime}-j}\right) \equiv Y_{\ell-1}$ for some $\ell$ with $0<\ell \leqq k^{\prime}$. By Sobolev's inequalities this also implies that $f(u(\tau)) \in Y_{\ell-1}$ and since $T$ maps $\mathscr{H}^{m}$ into $\mathscr{H}^{m-1}$ for any $m$, also $U(-\tau)$ 
$f(u(\tau)) \in Y_{\ell-1}$. From (2.11) written in the form

$$
\tilde{u}(t) \equiv U(-t) u(t)=u_{0}+\int_{0}^{t} d \tau U(-\tau) f(u(\tau))
$$

it follows that $\tilde{u} \in \mathscr{C}\left(I, \mathscr{H}^{k^{\prime}}\right) \cap\left(\bigcap_{0 \leqq j \leqq \ell-1} \mathscr{C}^{j+1}\left(I, \mathscr{H}^{k^{\prime}-j}\right)\right)$. That in turn implies that $u \in \mathscr{C}^{\ell}\left(I, \mathscr{H}^{k^{\prime}-\ell}\right)$, which completes the induction.

Remark 2.1. From the equations $\partial_{0} A_{j}=F_{0 j}$ and $\partial_{0} \phi=\psi_{0}$ and from Proposition 2.2, it follows that each component of $A_{j}$ and of $\phi$ actually belongs to $\mathscr{C}^{\ell+1}\left(I, H^{k^{\prime}-\ell}\right)$ for all $\ell, 0 \leqq \ell \leqq k^{\prime}$.

Remark 2.2. In the standard regularity theorems ([10], Theorem 3, p. 353, and corollaries), space regularity of the solutions follows from the fact that the solutions remain in the domain of suitable powers of the infinitesimal generator $T$ of the free group. In the present case however, $T$ is highly degenerate and these domains are not related in a simple way to the spaces $\mathscr{H}^{k}$, so that it is easier to give the previous direct proof.

So far we have considered the system (2.4) and (2.5) without taking into account the constraints (2.6), (2.7) and (2.8). We now show that these constraints are preserved in time.

Proposition 2.3. Let $k=[n / 2+1]$, let $V \in \mathscr{C}^{k+1}\left(\mathbb{R}^{+}\right)$and let $u_{0} \in \mathscr{H}^{k}$. Let I be an interval of $\mathbb{R}$ containing the origin, and let $u \in \mathscr{C}\left(I, \mathscr{H}^{k}\right)$ be a solution of $(2.11)$. Let $u_{0}$ satisfy (2.6) (respectively (2.8)) at $t=0$. Then $u(t)$ satisfies (2.6) (respectively (2.8)) for all $t \in I$. If in addition $u_{0}$ satisfies (2.7) at $t=0$, then $u(t)$ satisfies (2.7)for all $t \in I$.

Proof. From Proposition 2.2 and Remark 2.1 it follows that the quantities $F_{j k}$ $\partial_{j} A_{k}+\partial_{k} A_{j}-e\left[A_{j}, A_{k}\right]$ and $\psi_{j}-D_{j} \phi$ belong to $\mathscr{C}^{1}\left(I, H^{k-1}\right)$ componentwise. Furthermore their first time derivatives vanish by (2.4) and (2.5), so that they vanish for all times. Similarly from Proposition 2.2 it follows that $K_{0} \in \mathscr{C}^{1}\left(I, H^{k-2}\right)$ componentwise and that $K_{j} \in \mathscr{C}\left(I, H^{k-1}\right)$ componentwise. Therefore $D^{\mu} K_{\mu} \in$ $\mathscr{C}\left(I, H^{k-2}\right)$ and, by a direct computation, using (2.4), (2.5), (2.6) and (2.8), $D^{\mu} K_{\mu}=0$. This implies that $\partial_{0} K_{0}=0$, so that $K_{0}$ vanishes for all times.

Q.E.D.

\section{Abstract Cauchy Problem in Local Spaces for Small Time Intervals}

In this section we develop a general theory of equations of the type (2.11) in local spaces. In order to cover our needs and to illustrate the scope of the method, it will be sufficient to consider spaces of Sobolev type. Let therefore $X$ be a finite direct sum of Sobolev spaces $W^{m, p}\left(\mathbb{R}^{n}\right)$ with $m$ non-negative integer and $1 \leqq p<\infty$, possibly for different values of $m$ and $p$. For any open set $\Omega$ in $\mathbb{R}^{n}$, let $X(\Omega)$ be the corresponding direct sum of $W^{m, p}(\Omega)$. In this section we shall denote by $\|\cdot\|$ the norm in $X$ and by $\|\cdot\|_{\Omega}$ the norm in $X(\Omega)$. For any open set $\Omega$, let $r_{\Omega}$ be the operator of restriction to $\Omega$, namely the multiplication by the characteristic function of $\Omega$. For any open set $\Omega^{\prime} \supset \Omega, r_{\Omega}$ is a norm decreasing map from $X\left(\Omega^{\prime}\right)$ to $X(\Omega)$. For any 
open set $\Omega$, we define $X_{\text {loc }}(\Omega)$ as the space of functions $u$ such that $r_{\Omega^{\prime}} u \in X\left(\Omega^{\prime}\right)$ for any open bounded set $\Omega^{\prime}$ with closure $\bar{\Omega}^{\prime}$ contained in $\Omega\left(\Omega^{\prime} \subset \subset \Omega\right)$. $X_{\text {loc }}(\Omega)$ is a Frechet space, when equipped with the family of seminorms $\|u\|_{\Omega^{\prime}} \equiv\left\|r_{\Omega^{\prime}} u\right\|$. We shall use the notation $X_{\text {loc }}$ for $X_{\text {loc }}\left(\mathbb{R}^{n}\right)$. For any bounded open set $\Omega$ with regular boundary $\partial \Omega$ (by which we mean that $\partial \Omega$ is smooth, for instance $\mathscr{C}^{\infty}$, and that locally $\Omega$ lies on only one side of it), it is known [1] that there exists an extension $j_{\Omega}$ which is a bounded map from $X(\Omega)$ to $X$, namely

$$
\left\|j_{\Omega} u\right\| \leqq C_{1}(\Omega)\|u\|_{\Omega}
$$

and such that $r_{\Omega} j_{\Omega} u=u$ for all $u \in X(\Omega)$. Furthermore one can choose $j_{\Omega}$ such that for all $u \in X(\Omega), j_{\Omega} u$ has compact support contained in a fixed bounded open set $\Omega^{\prime} \supset \supset \Omega([1]$ Theorem 4.26, p.84). We shall be interested especially in the case where $\Omega$ is the open ball $B(x, R)$ with center $x$ and radius $R$ in $\mathbb{R}^{n}$. In this case we shall make the following choice for $j_{\Omega}$. For each component $u_{\sigma} \in W^{m, p}(B(0,1))$, we choose an extension $j_{1 \sigma}$ such that, for some $\delta>0$, Supp $j_{1 \sigma} u_{\sigma} \subseteq B(0,1+\delta)$ for all $u_{\sigma} \in W^{m, p}(B(0,1))$ and such that $([1]$, loc. cit.), for any multiindex $\alpha, 0 \leqq|\alpha| \leqq m$,

$$
\left\|\partial^{\alpha} j_{1 \sigma} u_{\sigma}\right\|_{p} \leqq C \sum_{\beta:|\beta| \leqq|\alpha|}\left\|\partial^{\beta} u_{\sigma}\right\|_{p, 1}
$$

where $\|\cdot\|_{p}$ denotes the norm in $L^{p}\left(\mathbb{R}^{n}\right)$ and $\|\cdot\|_{p, R}$ denotes the norm in $L^{p}(B(0, R))$. The extension $j_{1}$ is defined as the appropriate direct sum of the $j_{1, \sigma}$ 's. Then, for any $\Omega=B(x, R)$ we set

$$
j_{\Omega}=D(x, R) j_{1} D(x, R)^{-1},
$$

where $D(x, R)$ is defined by $(D(x, R) u)(y)=u((y-x) / R)$ and maps $X(B(0,1))$ onto $X(B(x, R))$.

Lemma 3.1. Let $\Omega=B(x, R)$ and let $j_{\Omega}$ be given by (3.3).Then

$$
\left\|j_{\Omega} u\right\| \leqq C_{1}(R)\|u\|_{\Omega},
$$

where, for any $R_{0}>0, C_{1}(R)$ is uniformly bounded for $R \geqq R_{0}$.

Proof. By translation invariance in $\mathbb{R}^{n}$ it will be sufficient to consider the case $x=0$. In addition it will be sufficient to prove (3.4) for each component $u_{\sigma}$ of $u$, $u_{\sigma} \in W^{m, p}(\Omega)$. For brevity we drop the index $\sigma$. From (3.3) it follows that

$$
\begin{aligned}
&\left\|D(0, R) \partial^{\alpha} j_{1} D(0, R)^{-1} u\right\|_{p} \\
& \leqq C \sum_{\beta:|\beta| \leqq|\alpha|}\left\|D(0, R) \partial^{\beta} D(0, R)^{-1} u\right\|_{p, R}
\end{aligned}
$$

for all $u \in W^{m, p}(B(0, R))$. Since $\partial^{\alpha} D(0, R)=R^{-|\alpha|} D(0, R) \partial^{\alpha},(3.5)$ yields

$$
\left\|\partial^{\alpha} j_{\Omega} u\right\|_{p} \leqq C \sum_{\beta:|\beta| \leqq|\alpha|}\left\|\partial^{\beta} u\right\|_{p, R} R^{|\beta|-|\alpha|}
$$

from which the Lemma follows.

Q.E.D.

An essential ingredient of the theory is the fact that the free group $U(t)$ describes propagation with velocity at most one. In order to exploit this property we intro- 
duce some definitions. For any open set $\Omega \subset \mathbb{R}^{n}$ and for any $t \in \mathbb{R}$, we define

and

$$
\Omega_{+}(t)=\Omega \cup\left(\bigcup_{x \in \Omega} B(x,|t|)\right)
$$

$$
\Omega(t)=\overline{\mathrm{C}\left\{(\mathrm{C} \bar{\Omega})_{+}(t)\right\}},
$$

where $C$ denotes the complement in $\mathbb{R}^{n}$. If $\Omega=B(x, R)$, then $\Omega_{ \pm}(t)=B(x, R \pm|t|)$ with the convention that $B(x, R)=\varnothing$ if $R \leqq 0$.

We shall call dependence domain any open subset $Q$ of $\mathbb{R}^{+} \times \mathbb{R}^{n}$ (this means in particular that $Q:\left(\{0\} \times \mathbb{R}^{n}\right)$ is open in $\left.\mathbb{R}^{n+1}\right)$ such that for any $(t, x) \in Q, Q$ contains the set $\left\{\left(t^{\prime}, x^{\prime}\right): t^{\prime} \geqq 0\right.$ and $\left.\left|x-x^{\prime}\right| \leqq t-t^{\prime}\right\}$.

This definition implies in particular that the sets

$$
Q(t) \equiv\left\{x: x \in \mathbb{R}^{n} \text { and }(t, x) \in Q\right\}
$$

are open and that there exists a function $\theta$ from $\mathbb{R}^{n}$ to $\mathbb{R}^{+}$such that

$$
Q=\{(t, x): 0 \leqq t<\theta(x)\},
$$

and that for all $x, x^{\prime} \in \mathbb{R}^{n}$,

$$
\left|\theta(x)-\theta\left(x^{\prime}\right)\right| \leqq\left|x-x^{\prime}\right|
$$

Conversely, any function $\theta$ from $\mathbb{R}^{n}$ to $\mathbb{R}^{+}$satisfying (3.10) defines a dependence domain through (3.9). Clearly any finite intersection and any union of dependence domains is a dependence domain.

We now abstract the crucial property of the free evolution in the following definition. We shall call hyperbolic semigroup a strongly continuous one parameter semigroup $U(t)$ in $X_{\mathrm{loc}}$ for which, for any $t \in \mathbb{R}^{+}$, for any open ball $\Omega=B(x, R)$, there exists a constant $\mu(\Omega, t) \geqq 0$, uniformly bounded for $(R, t)$ in compact subsets of $(0, \infty) \times[0, \infty)$, such that

$$
\|U(t) u\|_{\Omega_{-}(t)} \leqq \mu(\Omega, t)\|u\|_{\Omega}
$$

for any $u \in X_{\text {loc }}$.

The following lemma is an immediate consequence of the previous definition

Lemma 3.2. Let $t \in \mathbb{R}^{+}$and let $\Omega$ be an open ball in $\mathbb{R}^{n}$. Then

1) For any $u_{1}, u_{2} \in X_{\mathrm{loc}}$ such that $r_{\Omega+(t)} u_{1}=r_{\Omega+(t)} u_{2}$,

$$
r_{\Omega} U(t) u_{1}=r_{\Omega} U(t) u_{2} \text {. }
$$

2) Let $\Omega^{\prime}$ be a bounded open set containing $\Omega_{+}(t)$ (respectively an open set containing $\left.\Omega_{+}(t)\right), \Omega^{\prime \prime}$ a bounded open set with regular boundary such that $\Omega_{+}(t) \subset \Omega^{\prime \prime} \subset \Omega^{\prime}$ (respectively $\Omega_{+}(t) \subset \Omega^{\prime \prime} \subset \subset \Omega^{\prime}$ ) and $j_{\Omega^{\prime \prime}}$ an extension from $X\left(\Omega^{\prime \prime}\right)$ to $X$. Then

$$
U_{\Omega \Omega^{\prime}}(t) \equiv r_{\Omega} U(t) j_{\Omega^{\prime \prime}} r_{\Omega^{\prime \prime}}
$$

defines a bounded operator from $X\left(\Omega^{\prime}\right)$ to $X(\Omega)$ (respectively from $X_{\mathrm{loc}}\left(\Omega^{\prime}\right)$ to $X(\Omega)$ ) independent of $\Omega^{\prime \prime}$ and $j_{\Omega^{\prime \prime}}$. 
3) The operator $U_{\Omega \Omega^{\prime}},(t)$ is strongly continuous in $t$ for any $t$ such that $\Omega_{+}(t) \subset \subset \Omega^{\prime}$.

We now turn to the interaction term. We shall call local interaction a continuous function $f$ from $I \times X_{\text {loc }}$ to $X_{\text {loc }}$, where $I$ is an interval of $\mathbb{R}$ containing the origin, such that for any compact subinterval $J$ of $I$, for any open ball $\Omega$ in $\mathbb{R}^{n}$, for any $\rho \in \mathbb{R}^{+}$, the following inequality holds

$$
\operatorname{Sup}_{t \in J}\|f(t, u)-f(t, v)\|_{\Omega} \leqq C_{0}(\Omega, J, \rho)\|u-v\|_{\Omega}
$$

for all $u, v \in X_{\text {loc }}$ such that $\|u\|_{\Omega},\|v\|_{\Omega} \leqq \rho$. In addition for $\Omega=B(x, R), C_{0}(\Omega, J, \rho)$ is assumed to be bounded for $R$ in any compact subset of $(0, \infty)$.

The previous definition implies immediately the following lemma.

Lemma 3.3. Let $\Omega$ be an open ball in $\mathbb{R}^{n}$. Let $\Omega^{\prime}$ be a bounded open set containing $\Omega$ (respectively an open set containing $\bar{\Omega}), \Omega^{\prime \prime}$ a bounded open set with regular boundary such that $\Omega \subset \Omega^{\prime \prime} \subset \Omega^{\prime}$ (respectively $\Omega \subset \Omega^{\prime \prime} \subset \subset \Omega^{\prime}$ ), and $j_{\Omega^{\prime \prime}}$ an extension from $X\left(\Omega^{\prime \prime}\right)$ to $X$. Then

$$
f_{\Omega, \Omega^{\prime}}(t, u) \equiv r_{\Omega} f\left(t, j_{\Omega^{\prime \prime}} r_{\Omega^{\prime \prime}} u\right)
$$

defines a continuous map from $I \times X\left(\Omega^{\prime}\right)$ to $X(\Omega)$ (respectively from $I \times X_{\mathrm{loc}}\left(\Omega^{\prime}\right)$ to $X(\Omega)$ ), independent of $\Omega^{\prime \prime}$ and $j_{\Omega^{\prime \prime}}$.

Remark 3.1. The choice of the extension $j$ in Lemmas 3.2 and 3.3 is immaterial. The only role played by $j$ is to ensure that for any open set $\Omega^{\prime}$ and any open ball $\Omega \subset \subset \Omega^{\prime}$ the map $u \rightarrow r_{\Omega} u$ from $X_{\mathrm{loc}}\left(\Omega^{\prime}\right)$ to $X(\Omega)$ is surjective.

We shall look for solutions of the equation

$$
u(t)=U(t) u_{0}+\int_{0}^{t} d \tau U(t-\tau) f(\tau, u(\tau))
$$

where $U(t)$ is an hyperbolic semigroup, $f(t, u)$ is a local interaction, defined for $t \in I$, and $u_{0}$ is the initial data, in the following sense. Let $Q$ be a dependence domain such that $[0, \bar{t}) \subset I$, where $\bar{t}=\operatorname{Sup}\{t: Q(t) \neq \varnothing\}$. By an $X_{10 c}$-valued solution of (3.16) in $Q$, we mean a set of pairs $\{(t, u(t)\}, 0 \leqq t<\bar{t}$, such that

(1) for any $t \in[0, \bar{t}), u(t) \in X_{\mathrm{loc}}(Q(t))$,

(2) for any $t \in[0, \bar{t})$ and for any open ball $\Omega \subset \subset Q(t), r_{\Omega} u \in \mathscr{C}([0, t], X(\Omega))$ and the following equality holds

$$
\begin{aligned}
& r_{\Omega} u(t)=U_{\Omega, Q(0)}(t) u_{0} \\
& +\int_{0}^{t} d \tau U_{\Omega, \Omega_{+}(t-\tau)}(t-\tau) f_{\Omega_{+}(t-\tau), Q(t-\tau)}(\tau, u(\tau)) .
\end{aligned}
$$

It is clear that, if $Q$ and $Q^{\prime}$ are two dependence domains with $Q \subset Q^{\prime}$, any solution in $Q^{\prime}$ has a natural restriction to $Q$ which is a solution in $Q$.

We can now prove the following uniqueness result.

Proposition 3.1. Let $Q_{i}, i=1,2$, be two dependence domains, let $U(t)$ be an hyperbolic semigroup, let $f_{i}, i=1,2$, be two local interactions defined for $t \in I$ and such that 
for all $t \in I$

$$
g(t, u) \equiv f_{1}(t, u)-f_{1}(t, 0)=f_{2}(t, u)-f_{2}(t, 0),
$$

and

$$
f_{1}(t, 0)=f_{2}(t, 0) \text { in } Q_{1}(t) \cap Q_{2}(t),
$$

let $u_{0 i} \in X_{\mathrm{loc}}\left(Q_{i}(0)\right), i=1,2$, be such that $u_{01}=u_{02}$ in $Q_{1}(0) \cap Q_{2}(0)$ and let $u_{i}$, $i=1,2$, be $X_{\text {loc }}$-valued solutions of (3.16) in $Q_{i}$ with initial data $u_{0 i}$ and interactions $f_{i}$. Then $u_{1}=u_{2}$ in $Q_{1} \cap Q_{2}$.

Proof. It is sufficient to prove that for any $t$ and any open ball $\Omega \subset \subset Q_{1}(t) \cap$ $Q_{2}(t), r_{\Omega} u_{1}(t)=r_{\Omega} u_{2}(t)$. By taking the difference of the equations (3.17) for $u_{1}$ and $u_{2}$ and by using Lemma 3.2, Lemma 3.3 and (3.19), we obtain

$$
\begin{aligned}
& r_{\Omega_{+}(t-s)}\left(u_{1}(s)-u_{2}(s)\right)=\int_{0}^{s} d \tau U_{\Omega_{+}(t-s), \Omega_{+}(t-\tau)}(s-\tau) \\
& \quad\left\{g_{\Omega_{+}(t-\tau), Q_{1}(t-\tau)}\left(\tau, u_{1}(\tau)\right)-g_{\Omega_{+}(t-\tau), Q_{2}(t-\tau)}\left(\tau, u_{2}(\tau)\right)\right\}
\end{aligned}
$$

for all $s$ with $0 \leqq s \leqq t$. Lemmas 3.2 and 3.3 and (3.11), (3.14), then imply

$$
\left\|u_{1}(s)-u_{2}(s)\right\|_{\Omega_{+}(t-s)} \leqq C \int_{0}^{s} d \tau\left\|u_{1}(\tau)-u_{2}(\tau)\right\|_{\Omega_{+}(t-\tau)},
$$

where the constant $C$ depends on $\Omega$, on $t$, and on Max Sup $\left\|u_{i}(\tau)\right\|_{\Omega_{+}(t-\tau)}$. From (3.21) and the assumption $u_{01}=u_{02}$ in $Q_{1}(0) \cap Q_{2}(0)$ it follows that $u_{1}(s)=u_{2}(s)$ in $\Omega_{+}(t-s)$ for all $s \in[0, t]$.

Q.E.D.

We now turn to the existence problem. We first consider the case where $u_{0}$ and $f(t, 0)$ have compact support.

Proposition 3.2. Let $U(t)$ be an hyperbolic semigroup, let $\Omega$ be an open ball in $\mathbb{R}^{n}$, Let $f$ be a local interaction defined for $t \in I$ with Supp $f(t, 0) \subset \overline{\Omega_{+}(t)}$ for all $t \in I$, let $u_{0} \in X$ with Supp $u_{0} \subset \bar{\Omega}$. Then there exists a $T_{0}>0$ with $T_{0} \in I$ and a function $u \in \mathscr{C}\left(\left[0, T_{0}\right], X\right)$ with Supp $u(t) \subset \overline{\Omega_{+}(t)}$ for all $t \in\left[0, T_{0}\right]$ and satisfying $(3.16)$ for all $t \in\left[0, T_{0}\right]$.

Proof. For any $T>0, T \in I$, we define

$$
\mathscr{X}_{T}=\left\{u: u \in \mathscr{C}([0, T], X) \text { and } \operatorname{Supp} u(t) \subseteq \overline{\Omega_{+}(t)} \text { for all } t \in[0, T]\right\} .
$$

One sees easily that $\mathscr{X}_{T}$ is a Banach space when equipped with the norm

$$
\|u\|\left\|=\operatorname{Sup}_{t \in[0, T]}\right\| u(t) \|_{\Omega_{+}(t)} .
$$

The operator $S$, defined by

$$
(S u)(t) \equiv U(t) u_{0}+\int_{0}^{t} d \tau U(t-\tau) f(\tau, u(\tau))
$$


maps $\mathscr{X}_{T}$ into $\mathscr{X}_{T}$ and, for $T$ sufficiently small, is a contraction, as can be seen from (3.11) and (3.14). The fixed point of this contraction provides the solution we are looking for.

Q.E.D.

Remark 3.2. In the special case under consideration, Proposition 3.2 provides a solution of the equation (3.16) in the dependence domain

$$
Q=\left(\left[0, T_{0}\right) x \mathbb{R}^{n}\right) \cup\left\{(t, x): t \geqq 0 \text { and } x \notin \bar{\Omega}_{+}(t)\right\} .
$$

This solution is obtained by extending the function $u$ obtained in Proposition 3.2 by zero in the part of $Q$ with $t \geqq T_{0}$.

We are now able to treat the local existence problem for general initial data $u_{0} \in X_{\text {loc }}(\Omega)$ where $\Omega$ is an arbitrary open subset of $\mathbb{R}^{n}$ (possibly $\Omega=\mathbb{R}^{n}$ ).

Proposition 3.3. Let $U(t)$ be an hyperbolic semigroup, $f$ a local interaction defined for $t \in I$, let $\Omega$ be an open subset of $\mathbb{R}^{n}$, and let $u_{0} \in X_{\mathrm{loc}}(\Omega)$. Then there exists a dependence domain $Q$ with $Q(0)=\Omega$ (i.e the associated function $\theta$ is strictly positive in $\Omega$ ) and a (unique) $X_{\text {loc }}$-valued solution of (3.16) in $Q$.

Proof. We first show that to any open ball $\Omega_{0}=B\left(x_{0}, R_{0}\right) \subset \subset \Omega$ there is associated a (non-trivial) dependence domain $Q_{\Omega_{0}}$ with $Q_{\Omega_{0}}(0)=\Omega_{0}$, and a solution of (3.16) in $Q_{\Omega_{0}}$. In fact let $u_{0}^{\prime}=j_{\Omega_{0}} r_{\Omega_{0}} u_{0}$ and

$$
f^{\prime}(t, u)=j_{\Omega_{0}} r_{\Omega_{0}} f(t, 0)+f(t, u)-f(t, 0) .
$$

Then $u_{0}^{\prime}$ and $f^{\prime}(t, 0)$ have compact support contained in the ball $\Omega^{\prime}=$ $B\left(x_{0},(1+\delta) R_{0}\right)$. By Proposition (3.2), there exists $T_{\Omega_{0}}$ and $u_{\Omega_{0}} \in \mathscr{C}\left(\left[0, T_{\Omega_{0}}\right], X\right)$ satisfying (3.16) for all $t \in\left[0, T_{\Omega_{0}}\right]$ with $u_{0}$ replaced by $u_{0}^{\prime}$ and $f$ by $f^{\prime}$. Applying $r_{\Omega_{0}-}(t)$ to this equation for arbitrary $t \in\left[0, T_{\Omega_{0}}\right]$ one sees easily that the restriction of $u_{\Omega_{0}}$ to the dependence domain $Q_{\Omega_{0}}=\left\{(t, x): 0 \leqq t<T_{\Omega_{0}}, x \in \Omega_{0^{-}}(t)\right\}$ is a solution of (3.16) in that domain.

We now define $Q$ as the union of the dependence domains $Q_{\Omega_{0}}$ associated in this way to all open balls $\Omega_{0} \subset \subset \Omega$ and we define $u$ in $Q$ by $u=u_{\Omega_{0}}$ in $Q_{\Omega_{0}}$. This definition makes sense by Proposition 3.1 and $u$ thereby defined is easily seen to be an $X_{\text {loc }}$-valued solution of (3.16) in $Q$.

Q.E.D.

Remark 3.3. The use of the extension $j$ in the proof of Proposition 3.3 is not essential and can be avoided by a cutoff procedure. For $\Omega_{0}=B\left(x_{0}, R_{0}\right) \subset \subset \Omega$, let $\Omega^{\prime \prime}$ be an open ball with $\Omega_{0} \subset \subset \Omega^{\prime \prime} \subset \subset \Omega$, let $g \in \mathscr{C}_{0}^{\infty}$ with $0 \leqq g \leqq 1, g=1$ in $\Omega_{0}$ and $g=0$ in $\mathcal{C} \Omega^{\prime \prime}$, and let $u_{0}^{\prime \prime}=g u_{0}, f^{\prime \prime}(t, u)=g f(t, 0)+f(t, u)-f(t, 0)$. Then $u_{0}^{\prime \prime} \in X$, Supp $u_{0}^{\prime \prime} \subset \bar{\Omega}^{\prime \prime}$ and Supp $f^{\prime \prime}(t, 0) \subset \bar{\Omega}^{\prime \prime}$. One can then proceed as in the proof of Proposition 3.3 with $u_{0}^{\prime}$ replaced by $u_{0}^{\prime \prime}$ and $f^{\prime}(t, u)$ by $f^{\prime \prime}(t u)$. The advantage of using the extension $j$ is that $u_{0}^{\prime}$ is estimated in terms of $r_{\Omega_{0}} u_{0}$ only, through

$$
\left\|u_{0}^{\prime}\right\| \leqq C_{1}\left(R_{0}\right)\left\|u_{0}\right\|_{\Omega_{0}}
$$

whereas $u_{0}^{\prime \prime}$ is estimated in terms of $r_{\Omega^{\prime \prime}} u_{0}$ through

$$
\left\|u_{0}^{\prime \prime}\right\| \leqq C\left(\Omega_{0}, \Omega^{\prime \prime}\right)\left\|u_{0}\right\|_{\Omega^{\prime \prime}}
$$

This point will be important in some future applications. In addition the constant $C_{1}\left(R_{0}\right)$ is independent of $x_{0}$, whereas for fixed $R_{0}$, the constant $C\left(\Omega_{0}, \Omega^{\prime \prime}\right)$ in general 
blows up as a function of $x_{0}$ when the distance of $\Omega_{0}$ to the boundary of $\Omega$ (and therefore to the boundary of $\Omega^{\prime \prime}$ ) tends to zero.

Remark 3.4. The shape of the dependence domain obtained in the proof of Proposition 3.3 (we restrict our attention to the case where $u_{0} \in X_{\text {loc }}$ ) will depend on the constants $\mu(\Omega, t)$ appearing in (3.11), $C_{0}(\Omega, J, \rho)$ appearing in (3.14), on the local norms of $u_{0}$ in the spaces $X(\Omega)$ and, through the construction of $u_{0}^{\prime}$, on the constant $C_{1}(R)$ appearing in (3.4). Of special interest is the case when $\mu$ and $C_{0}$ are uniformly bounded with respect to space translations of $\Omega$. In this situation, if in addition $u_{0}$ is uniformly locally bounded, namely, if for some $R$ and $\Omega=B(x, R),\left\|u_{0}\right\|_{\Omega}$ is uniformly bounded in $x$, then, for $\Omega_{0}=B\left(x_{0}, R_{0}\right)$, the time $T_{\Omega_{0}}$ that occurs in the proof of Proposition 3.3 can be taken uniformly bounded from below with respect to $x_{0}, T_{\Omega_{0}} \geqq T_{0}>0$, so that the dependence domain $Q$ contains a strip $\left[0, T_{0}\right] \times \mathbb{R}^{n}$. This situation is considered in particular in [9]. If the local norm $\left\|u_{0}\right\|_{\Omega_{0}}$ increases when $\left|x_{0}\right|$ tends to infinity, we expect the dependence domain $Q$ to become thinner at infinity. On the contrary, if the local norm $\left\|u_{0}\right\|_{\Omega_{0}}$ tends to zero as $\left|x_{0}\right|$ tends to infinity, the dependence domain is expected to thicken at infinity. In particular, if the constant $C_{0}(\Omega, J, \rho)$ tends to zero when $\rho$ tends to zero for fixed $\Omega$ and $J$ and if the local norm of $u_{0}$ decreases sufficiently fast at infinity, one can ensure that the function $\theta(x)$ defining $Q$ (see (3.9) and (3.10)) increases linearly at infinity, namely $\theta(x) \sim \gamma|x|$ for some $\gamma, 0<\gamma<1$, thereby solving the so-called "boost problem" considered in a more general setting in [2]. This is achieved by imposing conditions on $u_{0}$ that ensure that, for any $\Omega_{0}=B\left(x_{0}, \gamma\left|x_{0}\right|\right)$ with $\left|x_{0}\right| \geqq a>0$, one can take $T_{\Omega_{0}}=\gamma\left|x_{0}\right|$, so that $Q$ contains the region $\{(t, x): 0 \leqq t<\gamma|x|\}$. In fact, it follows from (3.4), (3.11) and (3.14) that a sufficient condition for the operator $S$ defined by (3.24) with $u_{0}$ replaced by $u_{0}^{\prime}=j_{\Omega_{0}} r_{\Omega_{0}} u_{0}$ to be contracting in $\mathscr{X}_{T}$ is

$$
\begin{aligned}
& T \operatorname{Sup}_{\substack{0 \leqq t \leqq T \\
R \leqq L \leqq R+2 T}} \mu\left(B\left(x_{0}, L\right), t\right) \\
& \quad \times C_{0}\left(B\left(x_{0}, R+T\right),[0, T], C_{1}\left(\gamma\left|x_{0}\right|\right)\left\|u_{0}\right\|_{B\left(x_{0}, \gamma\left|x_{0}\right|\right)}\right)<1,
\end{aligned}
$$

where $R=(1+\delta) \gamma\left|x_{0}\right|$. For $T=\gamma\left|x_{0}\right|$ and if $C(\Omega, J, \rho)$ tends to zero as $\rho$ tends to zero, (3.25) takes the form

$$
\left\|u_{0}\right\|_{B\left(x_{0}, \gamma\left|x_{0}\right|\right)} \leqq \varphi\left(x_{0}\right)
$$

for a suitable function $\varphi$. This condition expresses the required decrease of $u_{0}$ at infinity. More precise estimates would require more detailed information on $U(t)$ and on $f(t, u)$. The method has a large flexibility and can handle more complicated situations, where for instance $u_{0}$ tends to zero in some directions, and to infinity in others, when $\left|x_{0}\right|$ tends to infinity. The dependence domain of Remark 3.2 is a limiting case of the previous construction: with the best possible decrease available, namely with $u_{0}$ having compact support, the dependence domain $Q$ has $\theta(x) \sim|x|$ at infinity.

\section{The Cauchy Problem in Local Spaces for Small Time Intervals}

In this section we apply the general theory developed in Sect. 3 to the coupled Yang-Mills and scalar fields previously considered in global spaces in Sect. 2. 
We consider again the system (2.4) and (2.5) in the integral form (2.11), supplemented by the constraints (2.6), (2.7) and (2.8). The space $X$ of Sect. 3 now becomes the space $\mathscr{H}^{k}$ of Sect. 2 and, for any open set $\Omega \subset \mathbb{R}^{n}$, the spaces $\mathscr{H}^{k}(\Omega)$ and $\mathscr{H}_{\text {loc }}^{k}(\Omega)$ are defined accordingly. In order to apply the general theory we have to verify that $U(t)$ defined by (2.12) and (2.13), is an hyperbolic semigroup and that $f$, which can be read off from (2.4) and (2.5), is a local interaction in the sense of Sect. 3. For this purpose it will be convenient to introduce the following notation: for any open ball $\Omega$, for any $t \in \mathbb{R}$, we define

$$
Q(\Omega, t)=\left\{\left(t^{\prime}, x^{\prime}\right): 0 \leqq\left|t^{\prime}\right|<|t|, t t^{\prime} \geqq 0 \text { and } x^{\prime} \in \Omega_{-}\left(t^{\prime}\right)\right\} .
$$

(For $t>0, Q(\Omega, t)$ is a dependence domain in the sense of Sect. 3.) In addition, for any $q, 1 \leqq q \leqq \infty$, for any open set $\Omega$, we denote by $\|\cdot\|_{q, \Omega}$ the norm in $L^{q}(\Omega)$.

Lemma 4.1. For any non-negative integer $k, U(t)$ is a strongly continuous oneparameter group in $\mathscr{H}_{\mathrm{loc}}^{k}$, and, for any $t \in \mathbb{R}$, for any open ball $\Omega \subset \mathbb{R}^{n}$, for any $u \in \mathscr{H}_{\mathrm{loc}}^{k}$, the following estimate holds:

$$
\|U(t) u\|_{\Omega_{-}(t)} \leqq \mu(t)\|u\|_{\Omega},
$$

where $\mu(t)$ is defined by (2.15). In particular $U(t)$ is an hyperbolic group in $\mathscr{H}_{\text {loc }}^{k}$.

Proof. It will be sufficient to prove (4.2) for $t$ positive and smooth $u$. The estimate is satisfied separately by $U_{A}(t)$ and $U_{\phi}(t)$. We prove it only for $U_{A}(t)$, the proof for $U_{\phi}(t)$ being similar. For any solution of the free evolution equation $u_{A}(t)=$ $U_{A}(t) u_{A}(0)$ (see (2.10)) it is well known and easy to check that for any multiindex $\alpha$ of space derivatives,

where

$$
\partial_{\mu} \Theta^{\mu}=0
$$

and

$$
\Theta^{0}=-\frac{1}{2}\left\langle\partial^{\alpha} F^{0 j}, \partial^{\alpha} F_{0 j}\right\rangle+\frac{1}{4}\left\langle\partial^{\alpha} F^{j \ell}, \partial^{\alpha} F_{j \ell}\right\rangle
$$

$$
\Theta^{\ell}=\left\langle\partial^{\alpha} F_{0 j}, \partial^{\alpha} F^{j \ell}\right\rangle
$$

The vector $\Theta^{\mu}$ is time-like by Schwarz's inequality and has a positive time component. Now let $\Omega$ be an open ball and $t>0$. Integrating (4.3) in $Q(\Omega, t)$, applying Gauss's theorem, and taking into account the fact that $\Theta^{\mu}$ is outgoing on the side surface of $Q(\Omega, t)$, we find

On the other hand

$$
\int_{\Omega_{-}(t)} d x \Theta^{0}(t) \leqq \int_{\Omega} d x \Theta^{0}(0)
$$

$$
\partial_{0}\left\{-\frac{1}{2}\left\langle\partial^{\alpha} A^{j}, \partial^{\alpha} A_{j}\right\rangle\right\}=-\left\langle\partial^{\alpha} A_{j}, \partial^{\alpha} F_{0 j}\right\rangle .
$$

If we now define

$$
M(t)^{2}=\sum_{1 \leqq j \leqq n}\left\|\left|\partial^{\alpha} F_{0 j}(t)\right|\right\|_{2, \Omega_{-}(t)}^{2}+\sum_{j<k}\left\|\left|\partial^{\alpha} F_{j k}(t)\right|\right\|_{2, \Omega_{-}(t)}^{2}
$$

and

$$
N(t)^{2}=\sum_{1 \leqq j \leqq n}\left\|\left|\partial^{\alpha} A_{j}(t)\right|\right\|_{2, \Omega_{-}(t)}^{2},
$$


then (4.6) and (4.7) yield

and

$$
M(t) \leqq M(0)
$$

$$
N(t) \leqq N(0)+\int_{0}^{t} d \tau M(\tau) \leqq N(0)+t M(0) .
$$

From there on the proof proceeds as that of Lemma 2.1.

We now turn to the interaction.

Q.E.D.

Lemma 4.2. Let $k \geqq[n / 2+1]$, let $V \in \mathscr{C}^{k+2}\left(\mathbb{R}^{+}\right)$and let $X=\mathscr{H}^{k}$. Then $f$ is a local interaction in $X_{1 \mathrm{loc}} \equiv \mathscr{H}_{\mathrm{loc}}^{k}$; more precisely, for any open ball $\Omega \subset \mathbb{R}^{n}$, for any $\rho \in \mathbb{R}^{+}$, $f$ satisfies the estimate

$$
\|f(u)-f(v)\|_{\Omega} \leqq C_{0}(\Omega, \rho)\|u-v\|_{\Omega}
$$

for all $u$ and $v \in \mathscr{H}_{\text {loc }}^{k}$ with $\|u\|_{\Omega},\|v\|_{\Omega} \leqq \rho$. Furthermore, for $\Omega=B(x, R), C_{0}(\Omega, \rho)$ can be taken independent of $x$ and uniformly bounded for $R \geqq R_{0}>0$.

Proof. The estimate (4.12) is a local version of the estimate (2.22) and follows from the assumptions on $V$, the fact that the terms in $f$ not containing $V$ are bilinear in the components of $u$ and from Sobolev inequalities in $\Omega$. These inequalities are derived from the corresponding ones in $\mathbb{R}^{n}$ by using the extension $j_{\Omega}$. The stated properties of $C_{0}(\Omega, \rho)$ follow from Lemma 3.1 .

The local existence result of Proposition 3.3 can be restated as follows.

Proposition 4.1. Let $k=[n / 2+1], V \in \mathscr{C}^{k+2}\left(\mathbb{R}^{+}\right)$and $u_{0} \in \mathscr{H}_{\text {loc }}^{k}$. Then there exists a dependence domain $Q$ with $Q(0)=\mathbb{R}^{n}$ and a (unique) $\mathscr{H}_{1 \mathrm{oc}}^{k}$-valued solution of (2.11). in $Q$ (in the sense of Sect. 3).

As compared to the general case, we note that here $\mu$ does not depend on $\Omega$ (compare (3.11) with (4.2)) and $C_{0}$ does not depend on $J$ (compare (3.14) with (4.12)) and can be taken invariant by translation of $\Omega$. As mentioned in Remark 3.2, this implies in particular that, if $u_{0}$ is uniformly locally bounded, then the dependence domain $Q$ of Proposition 4.1 contains a strip.

We now turn to the problem of the regularity of the solutions for suitably regular initial data.

Proposition 4.2. Let $k^{\prime} \geqq k=[n / 2+1]$, let $V \in \mathscr{C}^{k^{\prime}+1}\left(\mathbb{R}^{+}\right)$, let $Q$ be a dependence domain, let $u_{0} \in \mathscr{H}_{\mathrm{loc}}^{k^{\prime}}(Q(0))$ and let $u$ be an $\mathscr{H}_{\text {loc }}^{k}$-valued solution of $(2.11)$ in $Q$. Then, for any $t>0$ and any open ball $\Omega$ such that $\Omega \subset \subset Q(t), r_{\Omega} u \in \mathscr{C}^{\ell}\left([0, t], \mathscr{H}^{k^{\prime}-\ell}(\Omega)\right)$ for any $\ell, 0 \leqq \ell \leqq k^{\prime}$. In particular $u$ is an $\mathscr{H}_{\text {loc }}^{k^{\prime}}$-valued solution of (2.11) in $Q$.

Proof. Let $t>0$ and let $\Omega$ be an open ball such that $\Omega \subset \subset Q(t)$. Let $Q_{0}=Q\left(\Omega_{+}(t), t\right)$ (see (4.1)). We shall prove that there exists a finite covering of the interval $[0, t]$ by open subintervals $I_{j}$ of $\mathbb{R}^{+}$, and for each $j$, a function $u_{j} \in \mathscr{C}\left(I_{j}, \mathscr{H}^{k}\right)$ such that

(a) $u_{j}$ coincides with $u$ in $Q_{0} \cap\left(I_{j} \times \mathbb{R}^{n}\right)$,

(b) $u_{j} \in \mathscr{C}^{\ell}\left(I_{j}, \mathscr{H}^{k^{\prime}-\ell}\right)$ for any $\ell, 0 \leqq \ell \leqq k^{\prime}$.

Let $s \in[0, t]$ and let

$$
u^{\prime}(s)=j_{\Omega_{+}(t-s)} r_{\Omega_{+}(t-s)} u(s)
$$


where the extension $j_{\Omega}$ is adapted to the space $\mathscr{H}^{k^{\prime}}$. Then by Proposition 2.1, the equation

$$
v(t)=U(t-s) u^{\prime}(s)+\int_{s}^{t} d \tau U(t-\tau) f(v(\tau))
$$

has a unique solution $v_{s} \in \mathscr{C}\left(\left[s, s+T_{0}\right], \mathscr{H}^{k}\right)$. From Lemma 3.1 and the fact that

$$
\operatorname{Sup}_{0 \leqq s \leqq t}\|u(s)\|_{\Omega_{+}(t-s)}<\infty,
$$

it follows that $u^{\prime}(s)$ is uniformly bounded in $\mathscr{H}^{k}$ for $s \in[0, t]$ and therefore, by Proposition 2.1, $T_{0}$ can be taken independent of $s$. We now define $t_{j}=j T_{0} / 2$ for $0 \leqq j \leqq\left[2 t / T_{0}-1\right], I^{\prime}{ }_{j}=\left[t_{j}, t_{j}+T_{0}\right)$ and $u_{j}=v_{t_{j}}$. Then, for all $j, u_{j}$ solves the equation for $v$

$$
\begin{aligned}
r_{\Omega_{+}(t-s)} v(s)= & U_{\Omega_{+}(t-s), \Omega_{+}\left(t-t_{l}\right)} u^{\prime}\left(t_{j}\right) \\
& +\int_{t_{j}}^{s} d \tau U_{\Omega_{+}(t-s), \Omega_{+}(t-\tau)} f_{\Omega_{+}(t-\tau), Q(t-\tau)}(v(\tau))
\end{aligned}
$$

for all $s \in I_{j}^{\prime}$. On the other hand, it follows from (3.17) and (4.13) that $u$ also solves (4.16) in $I_{j}^{\prime}$ for all $j$. The same argument as in the proof of Proposition 3.1 then implies that $u=u_{j}$ in the translated dependence domain $Q_{0} \cap\left(I_{j}^{\prime} \times \mathbb{R}^{n}\right)$. This proves statement (a) above with $I_{0}=I_{0}^{\prime}$ and $I_{j}=\left(t_{j}, t_{j}+T_{0}\right)$ for $1 \leqq j \leqq\left[2 t / T_{0}-1\right]$. Statement (b) follows from Proposition 2.2 by an elementary induction argument on $j$. Proposition 4.2 is an immediate consequence of statements (a) and (b).

Q.E.D.

Remark 4.1. From the equations $\partial_{0} A_{j}=F_{0 j}$ and $\partial_{0} \phi=\psi_{0}$, it follows that for any $t>0$ and any open ball $\Omega$ such that $\Omega \subset \subset Q(t)$, each component of $A_{j}$ and of $\phi$ actually belongs to $\mathscr{C}^{\ell+1}\left([0, t], H^{k^{\prime}-\ell}(\Omega)\right)$ for all $\ell, 0 \leqq \ell \leqq k^{\prime}$.

As in Sect. 2, we now show that the constraints (2.6), (2.7) and (2.8) are preserved in time for the solutions of (2.11).

Proposition 4.3. Let $k=[n / 2+1]$, let $V \in \mathscr{C}^{k+1}\left(\mathbb{R}^{+}\right)$, let $Q$ be a dependence domain, let $u_{0} \in \mathscr{H}_{\text {loc }}^{k}(Q(0))$ and let $u$ be an $\mathscr{H}_{\text {loc }}^{k}$-valued solution of (2.11) in $Q$. Let $u_{0}$ satisfy (2.6) (respectively (2.8)) in $Q(0)$. Then $u(t)$ satisfies (2.6) (respectively (2.8)) in $Q(t)$ for all $t$. If in addition $u_{0}$ satisfies (2.7), then $u(t)$ satisfies (2.7) in $Q(t)$ for all $t$.

Proof. The proof is similar to that of Proposition 2.3. One simply replaces the spaces $\mathscr{C}^{\ell}\left(I, H^{m}\right)$ by $\mathscr{C}^{\ell}\left([0, t], H^{m}(\Omega)\right)$ with $t>0$ and $\Omega$ an open ball such that $\Omega \subset \subset Q(t)$. In the case where $m<0$ (this occurs for the preservation of (2.7) for $n=1$ ), one uses instead of $H^{m}(\Omega)$ the space of distributions $\mathscr{D}^{\prime}\left(\Omega_{1}\right)$ for some open ball $\Omega_{1}$ such that $\Omega \subset \subset \Omega_{1} \subset \subset Q(t)$.

Q.E.D.

\section{Existence of Global Solutions}

In this section we prove the existence of global solutions of the equation (2.11) for $n=1,2$, and make some comments on the cases $n=3,4$. The proof relies on a priori bounds on the solutions in $\mathscr{H}^{k}$. These in turn follow from estimates on 
conserved or quasiconserved quantities which we now set out to define formally. The energy of the system is defined by

$$
\begin{aligned}
E_{0}= & \int d x\left\{-\frac{1}{2}\left\langle F^{0 j}, F_{0 j}\right\rangle+\frac{1}{4}\left\langle F^{j \ell}, F_{j \ell}\right\rangle\right. \\
& \left.+\left\langle\psi^{0}, \psi_{0}\right\rangle-\left\langle\psi^{j}, \psi_{j}\right\rangle+V\left(|\phi|^{2}\right)\right\} .
\end{aligned}
$$

More generally we define

$$
\begin{gathered}
E_{1}=\int d x\left\{\frac{1}{2}\left\langle D^{\ell} F^{0 j}, D_{\ell} F_{0 j}\right\rangle-\frac{1}{4}\left\langle D^{\ell} F^{i j}, D_{\ell} F_{i j}\right\rangle\right. \\
\left.-\left\langle D^{\ell} \psi^{0}, D_{\ell} \psi_{0}\right\rangle+\left\langle D^{\ell} \psi^{j}, D_{\ell} \psi_{j}\right\rangle\right\}, \\
E_{2}=\int d x\left\{-\frac{1}{2}\left\langle D^{k} D^{\ell} F^{0 j}, D_{k} D_{\ell} F_{0 j}\right\rangle+\frac{1}{4}\left\langle D^{k} D^{\ell} F^{i j}, D_{k} D_{\ell} F_{i j}\right\rangle\right. \\
\left.+\left\langle D^{k} D^{\ell} \psi^{0}, D_{k} D_{\ell} \psi_{0}\right\rangle-\left\langle D^{k} D^{\ell} \psi^{j}, D_{k} D_{\ell} \psi_{j}\right\rangle\right\},
\end{gathered}
$$

and so on. We shall derive the relevant estimates in Lemmas 5.1 to 5.6 below.

Lemma 5.1. Let $k=[n / 2+1]$, let $V \in \mathscr{C}^{k+1}\left(\mathbb{R}^{+}\right)$and let $u_{0} \in \mathscr{H}^{k}$. Let I be an interval of $\mathbb{R}$ containing the origin and let $u \in \mathscr{C}\left(I, \mathscr{H}^{k}\right)$ be a solution of $(2.11)$. Then

(1) $E_{0}(t)=E_{0}(0)$ for all $t \in I$.

(2) Let in addition $V$ satisfy the condition

$$
V(\rho) \geqq-a^{2} \rho
$$

for some $a \geqq 0$. Then $F_{0}, F, A, \psi_{0}, \psi$ and $\phi$ are estimated in $L^{2}$ in terms of the initial data for all $t \in I$. More precisely

$$
\begin{aligned}
& \frac{1}{2}\left\{\sum_{j}\left\|\left|F_{0 j}(t)\right|\right\|_{2}^{2}+\sum_{j<k}\left\|\left|F_{j k}(t)\right|\right\|_{2}^{2}\right\}+\left\|\left|\psi_{0}(t)\right|\right\|_{2}^{2} \\
& +\sum_{j}\left\|\left|\psi_{j}(t)\right|\right\|_{2}^{2} \leqq \bar{E}_{0}(t) \leqq\left(\frac{d v(t)}{d t}\right)^{2} \\
& \|\| \phi(t)\left|\left\|_{2} \leqq\right\|\right| \phi(0) \mid \|_{2}+v(t), \\
& \left\{\frac{1}{2} \sum_{j}\left\|\left|A_{j}(t)\right|\right\|_{2}^{2}\right\}^{1 / 2} \leqq\left\{\frac{1}{2} \sum_{j}\left\|\left|A_{j}(0)\right|\right\|_{2}^{2}\right\}^{1 / 2}+v(t),
\end{aligned}
$$

where

$$
\bar{E}_{0}(t)=E_{0}+a^{2}\||\phi(t)|\|_{2}^{2}
$$

and

$$
v(t)=\||\phi(0)|\|_{2}(\cosh a t-1)+\bar{E}_{0}(0)^{1 / 2} a^{-1} \sinh a|t| .
$$

Proof. To prove part (1), we remark that by Proposition 2.2, $u \in \mathscr{C}^{1}\left(I, \mathscr{H}^{k-1}\right)$ and therefore that $E_{0}(t)$ is differentiable in time. Taking its derivative and using the equation of motion (2.9), we see that $E_{0}(t)$ is constant in time.

To prove part (2), we note that

$$
\begin{aligned}
\partial_{0} \bar{E}_{0}(t) & =2 a^{2} \operatorname{Re}\left\langle\phi(t), \psi_{0}(t)\right\rangle \\
& \leqq 2 a^{2}\||\phi(t)|\|_{2} \bar{E}_{0}(t)^{1 / 2},
\end{aligned}
$$


where we have used (5.4) in the last inequality. By an elementary computation, this yields (5.5) and (5.6). The inequality (5.7) follows from (5.5) and from

$$
\partial_{0}\left\langle A^{j}, A_{j}\right\rangle=2\left\langle A^{j}, F_{0 j}\right\rangle \text {. }
$$

Q.E.D.

In order to simplify the notation, from now on we shall omit the $\mid$ when appearing in an $L^{p}$-norm, we shall denote generically $F_{\lambda, \mu}$ by $F$ and $\psi_{\mu}$ by $\psi$, and we shall drop the space-time indices in most of the estimates.

We now turn to the quantity $E_{1}$.

Lemma 5.2. Let $k=[n / 2+1]$, let $V \in \mathscr{C}^{k+2}\left(\mathbb{R}^{+}\right)$, and let $u_{0} \in \mathscr{H}^{k}$ satisfy the constraints (2.6) and (2.8). Let $I$ be an interval of $\mathbb{R}$ containing the origin and let $u \in \mathscr{C}\left(I, \mathscr{H}^{k}\right)$ be a solution of $(2.11)$. Then

(1) $E_{1}(t)$ is differentiable for all $t \in I$ and satisfies

$$
\begin{aligned}
\partial_{0} E_{1}= & \int d x\left\{e \left(\left\langle D_{\ell} F^{0 j},\left[F^{\mu \ell}, F_{\mu j}\right]\right\rangle\right.\right. \\
& \left.-\left\langle D_{\ell} F^{i j},\left[F^{0 \ell}, F_{i j}\right]\right\rangle\right)+2 e \operatorname{Re}\left(-\left\langle D_{\ell} \psi_{0}, F^{\mu \ell} \psi_{\mu}\right\rangle\right. \\
& +\left\langle D_{\ell} \psi_{j}+D_{j} \psi_{\ell}, F^{0 j} \psi^{\ell}\right\rangle+\left\langle D_{\ell} \psi_{j}, F^{\ell j} \psi_{0}\right\rangle \\
& \left.\left.-\left\langle\psi_{j},\left(D_{\ell} F^{0 j}\right) \psi^{\ell}\right\rangle\right)+2 \operatorname{Re}\left\langle D^{j} \psi_{0}, D_{j}\left(\phi V^{\prime}\right)\right\rangle\right\} .
\end{aligned}
$$

In addition let $V$ satisfy (5.4) and define the function $\varphi_{1}$ by

$$
\varphi_{1}(\rho)=\operatorname{Sup}_{0 \leqq \sigma \leqq \rho}\left(\left|V^{\prime}(\sigma)\right|+2 \sigma\left|V^{\prime \prime}(\sigma)\right|\right) .
$$

(2) Let $1 \leqq n \leqq 3$. For any $t \in I, E_{1}(t)$ satisfies the estimate

$$
\begin{aligned}
\left|\partial_{0} E_{1}(t)\right| \leqq & C\left\{E_{1}(t)^{1 / 2+n / 4} \bar{E}_{0}(t)^{1-n / 4}\right. \\
& \left.+E_{1}(t)^{1 / 2} \bar{E}_{0}(t)^{1 / 2} \varphi_{1}\left(\|\phi(t)\|_{\infty}^{2}\right)\right\}
\end{aligned}
$$

with

$$
\|\phi(t)\|_{\infty} \leqq \begin{cases}C\|\phi(t)\|_{2}^{1 / 2} \bar{E}_{0}(t)^{1 / 4} & (n=1) \\ C_{\varepsilon}\|\phi(t)\|_{2}^{\varepsilon / 2} \bar{E}_{0}(t)^{(1-\varepsilon) / 2} E_{1}(t)^{\varepsilon / 4} & (n=2) \\ C \bar{E}_{0}(t)^{1 / 4} E_{1}(t)^{1 / 4} & (n=3)\end{cases}
$$

and $0<\varepsilon<1$ for $n=2$.

In particular, for $n=1, E_{1}(t)$ is estimated in terms of the initial data for all $t \in I$.

(3) Let $n=2$ and let $V$ satisfy

$$
\varphi_{1}(\rho) \leqq b_{1}\left(1+\rho^{p}\right)
$$

for some $p, 0 \leqq p<\infty$. Then, for all $t \in I, E_{1}(t)$ satisfies the estimate

$$
\begin{aligned}
\left|\partial_{0} E_{1}(t)\right| \leqq & C\left\{E_{1}(t) \bar{E}_{0}(t)^{1 / 2}+E_{1}(t)^{1 / 2} \bar{E}_{0}(t)^{1 / 2}\right. \\
& \left.\times\left(1+E_{1}(t)^{\varepsilon / 2}\|\phi(t)\|_{2} \bar{E}_{0}(t)^{p-\varepsilon}\right)\right\}
\end{aligned}
$$

(with $0<\varepsilon<p$ ) for $p \geqq 1$, and a similar estimate for $p \leqq 1$. In particular, $E_{1}(t)$ is estimated in terms of the initial data for all $t \in I$. 
(4) Let $n=3,4$ and let $V$ satisfy (5.15) for some $p, 0 \leqq p<\infty$. Then, for all $t \in I$, $E_{1}(t)$ satisfies the estimate

$$
\begin{aligned}
\left|\partial_{0} E_{1}(t)\right| \leqq & C\left\{E_{1}(t)^{1 / 2+n / 4} \bar{E}_{0}(t)^{1-n / 4}\right. \\
& \left.+E_{1}(t)^{1 / 2} \bar{E}_{0}(t)^{1 / 2}\left(1+\left(E_{1}(t)^{n / 2-1} \bar{E}_{0}(t)^{2-n / 2}\right)^{p}\right)\right\}
\end{aligned}
$$

Proof. (1). It will be sufficient to prove the result for $u_{0} \in \mathscr{H}^{k+1}$. The general case where $u_{0} \in \mathscr{H}^{k}$ will follow from the fact that each $u_{0} \in \mathscr{H}^{k}$ satisfying the constraints (2.6) and (2.8) can be approximated by elements of $\mathscr{H}^{k+1}$ still satisfying the constraints (2.6) and (2.8), from the continuity of the solution of (2.11) with respect to the initial data $u_{0}\left([10]\right.$ Corollary 1.5, p. 350) and from the fact that both $E_{1}$ and the right hand side of (5.11) are continuous functions of $u \in \mathscr{H}^{k}$. For $u_{0} \in \mathscr{H}^{k+1}$, the solution $u(t)$ of $(2.11)$ belongs to $\mathscr{C}^{1}\left(I, \mathscr{H}^{k}\right)$ by Proposition 2.2 and satisfies the constraints (2.6) and (2.8) by Proposition 2.3. Therefore $E_{1}(t)$ is differentiable in $t$. Taking its derivative, using the equations of motion (2.9) and the constraints (2.6) and (2.8), we obtain (5.11). (The constraints are used to express the commutators of covariant space derivatives in terms of $F$ ).

(2), (3) and (4). We estimate the right hand side of (5.11). For brevity we omit the $t$ dependence in all this proof. The terms of order $e$ in the integrand are of the form $\langle D F,[F, F]\rangle,\langle\psi,(D F) \psi\rangle$ and $\langle D \psi, F \psi\rangle$. All these terms are estimated in the same way and we consider only the first one. Using the covariant Sobolev inequalities $(A .2)$, we obtain for $1 \leqq n \leqq 4$

$$
\begin{aligned}
\int d x|\langle D F,[F, F]\rangle| & \leqq C\|D F\|_{2}\|F\|_{4}^{2} \\
& \leqq C\|D F\|_{2}^{1+n / 2}\|F\|_{2}^{2-n / 2} \\
& \leqq C E_{1}^{1 / 2+n / 4} \bar{E}_{0}^{1-n / 4} .
\end{aligned}
$$

We next estimate the terms containing $V$ in the right hand side of (5.11). From the relation

$$
D_{j}\left(\phi V^{\prime}\right)=V^{\prime} \psi_{j}+2 V^{\prime \prime} \operatorname{Re}\left\langle\phi, \psi_{j}\right\rangle \phi
$$

it follows that

$$
\left|D_{j}\left(\phi V^{\prime}\right)\right| \leqq \varphi_{1}\left(|\phi|^{2}\right)\left|\psi_{j}\right|
$$

pointwise, and therefore

$$
\begin{aligned}
& \int d x\left|\left\langle D^{j} \psi_{0}, D_{j}\left(\phi V^{\prime}\right)\right\rangle\right| \leqq\|D \psi\|_{2}\left\|\varphi_{1}\left(|\phi|^{2}\right) \psi\right\|_{2} \\
& \quad \leqq C\|D \psi\|_{2}\|\psi\|_{2} \varphi_{1}\left(\|\phi\|_{\infty}^{2}\right),
\end{aligned}
$$

which together with (5.18) and its analogue for the terms of order $e$ containing $\psi$, implies (5.13).

On the other hand, for $1 \leqq n \leqq 3$, the covariant Sobolev inequalities, together with the constraint (2.8), imply that the $L^{\infty}$-norm of $\phi$ is estimated by

$$
\|\phi\|_{\infty} \leqq \begin{cases}C\|\phi\|_{2}^{1 / 2}\|\psi\|_{2}^{1 / 2} & (n=1) \\ C_{\varepsilon}\|\phi\|_{2}^{\varepsilon / 2}\|\psi\|_{2}^{1-\varepsilon}\|D \psi\|_{2}^{\varepsilon / 2} & (n=2) \\ C\|\psi\|_{2}^{1 / 2}\|D \psi\|_{2}^{1 / 2} & (n=3)\end{cases}
$$

which together with the definitions of $\bar{E}_{0}$ and $E_{1}$ yields (5.14). The proof of part (2) 
of the Lemma is completed by noting that the last statement follows from Lemma 5.1 , part (2), and from the fact that the right hand side of (5.13) is at most linear in $E_{1}$ for $n=1$.

In order to prove part (3), we note that (5.16) follows from (5.13), from (5.15) and from (5.14) with $\varepsilon$ replaced by $\varepsilon / p$ if $p \geqq 1$. A similar (actually better) estimate holds for $p \leqq 1$. The last statement of part (3) follows from Lemma 5.1, part (2), and from the fact that for $\varepsilon \leqq 1$ the right hand side of (5.16) is at most linear in $E_{1}$.

We finally turn to part (4). For $n=3$, the estimate (5.17) follows immediately from (5.13), (5.14) and (5.15). However this proof does not apply to the case $n=4$, since in that case $\phi$ is not estimated in $L^{\infty}$ in terms of $\bar{E}_{0}$ and $E_{1}$. We therefore give a direct proof (which also covers the case $n=3$ ). The contribution of the constant term in (5.15) to the right hand side of (5.20) is estimated by $\|\psi\|_{2}\|D \psi\|_{2}$. The contribution of the term $\rho^{p}$ is estimated as

$$
C\|D \psi\|_{2}\left\|\psi|\phi|^{2 p}\right\|_{2} \leqq C\|D \psi\|_{2}\|\psi\|_{r}\|\phi\|_{2 p q}^{2 p}
$$

by Hölder's inequality, with $1 / r+1 / q=1 / 2$. We take $1 / q=\varepsilon / n$ with $\varepsilon=$ Min $(1, p(n-2))$. Applying the covariant Sobolev inequalities in the form

$$
\begin{gathered}
\|\psi\|_{r} \leqq C\|D \psi\|_{2}^{\varepsilon}\|\psi\|_{2}^{1-\varepsilon}, \\
\|\phi\|_{2 p q}^{2 p} \leqq C\|D \psi\|_{2}^{p(n-2)-\varepsilon}\|\psi\|_{2}^{p(4-n)+\varepsilon},
\end{gathered}
$$

we estimate the last member of (5.23) as

$$
\begin{aligned}
\ldots & \leqq C\|D \psi\|_{2}^{1+p(n-2)}\|\psi\|_{2}^{1+p(4-n)} \\
& \leqq C E_{1}^{1 / 2+p(n / 2-1)} \bar{E}_{0}^{1 / 2+p(2-n / 2)} .
\end{aligned}
$$

Together with (5.18) and its analogues for the terms containing $\psi,(5.24)$ implies (5.17), thereby completing the proof of part (4) of the Lemma.

Q.E.D.

Remark 5.1. For space time dimensions 4 and 5, i.e. for $n=3$ and 4 , the right hand side of (5.18) increases faster than linearly in $E_{1}$, thereby precluding an a priori estimate of $E_{1}$ in terms of the initial data. However, from the covariant Sobolev inequalities, one obtains, for $2 \leqq n \leqq 4$,

$$
\int d x|\langle D F,[F, F]\rangle| \leqq C\|D F\|_{2}^{2}\|F\|_{n} .
$$

Similarly, the terms of order $e$ containing $\psi$ in the right hand side of (5.11) are estimated, possibly after shifting the covariant derivative from $F$ to $\psi$ by an integration by parts, as

$$
\int d x|\langle D \psi, F \psi\rangle| \leqq C\|D \psi\|_{2}^{2}\|F\|_{n} .
$$

From (5.25), (5.26) and from (5.24) it follows that an a priori estimate of $F$ in $L^{n}$ implies an a priori estimate of $E_{1}$, provided $V$ satisfies $(5.15)$ with $p \leqq(n-2)^{-1}$.

We now turn to the quantity $E_{2}$.

Lemma 5.3. Let $n \geqq 2, k=[n / 2+1]$, let $V \in \mathscr{C}^{k+2}\left(\mathbb{R}^{+}\right)$. Let $u_{0} \in \mathscr{H}^{k}$ satisfy the constraints (2.6) and (2.8). Let $I$ be an interval of $\mathbb{R}$ containing the origin and let $u \in \mathscr{C}\left(I, \mathscr{H}^{k}\right)$ be a solution of $(2.11)$. Then 
(1) $E_{2}(t)$ is differentiable for all $t \in I$ and satisfies

$$
\begin{aligned}
\partial_{0} E_{2}= & \int d x\left\{e O \left(\left\langle D^{2} F,[D F, F]\right\rangle+\left\langle D^{2} \psi, F D \psi\right\rangle\right.\right. \\
& \left.+\left\langle D^{2} \psi,(D F) \psi\right\rangle+\left\langle D \psi,\left(D^{2} F\right) \psi\right\rangle\right) \\
& \left.-2 \operatorname{Re}\left\langle D^{j} D^{\ell} \psi_{0}, D_{j} D_{\ell}\left(\phi V^{\prime}\right)\right\rangle\right\} .
\end{aligned}
$$

The part of order e in (5.27) is a sum of several terms of the form indicated with various combinations of indices.

Now in addition let $V$ satisfy (5.4), define $\varphi_{1}$ by (5.12) and $\varphi_{2}$ by

$$
\varphi_{2}(\rho)=\operatorname{Sup}_{0 \leqq \sigma \leqq \rho}\left(3 \sigma^{1 / 2}\left|V^{\prime \prime}(\sigma)\right|+2 \sigma^{3 / 2}\left|V^{\prime \prime \prime}(\sigma)\right|\right)
$$

(2) Let $n=2$. Then for all $t \in I, E_{2}(t)$ satisfies the estimate

$$
\begin{aligned}
\left|\partial_{0} E_{2}(t)\right| \leqq & C\left\{E_{2}(t)^{(1+\varepsilon) / 2} E_{1}(t)^{1-\varepsilon} \bar{E}_{0}(t)^{\varepsilon / 2}\right. \\
& \left.+E_{2}(t)^{1 / 2} E_{1}(t)^{1 / 2}\left(\varphi_{1}\left(\|\phi(t)\|_{\infty}^{2}\right)+\bar{E}_{0}(t)^{1 / 2} \varphi_{2}\left(\|\phi(t)\|_{\infty}^{2}\right)\right)\right\}
\end{aligned}
$$

with $0<\varepsilon<1$. In addition let $V$ satisfy $(5.15)$ for some $p, 0 \leqq p<\infty$. Then $E_{2}(t)$ is estimated in terms of the initial data for all $t \in I$.

(3) Let $n=3,4$. Then for all $t \in I, E_{2}(t)$ satisfies the estimate

$$
\begin{aligned}
\left|\partial_{0} E_{2}(t)\right| \leqq & C\left\{E_{2}(t)^{n / 4} E_{1}(t)^{3 / 2-n / 4}\right. \\
& +E_{2}(t)^{1 / 2}\left(E_{1}(t)^{1 / 2} \varphi_{1}\left(\|\phi(t)\|_{\infty}^{2}\right)\right. \\
& \left.\left.+E_{1}(t)^{n / 4} \bar{E}_{0}(t)^{1-n / 4} \varphi_{2}\left(\|\phi(t)\|_{\infty}^{2}\right)\right)\right\},
\end{aligned}
$$

with $\phi$ satisfying the estimate (5.14) for $n=3$ and

$$
\|\phi(t)\|_{\infty} \leqq C_{\varepsilon} \bar{E}_{0}(t)^{\varepsilon / 4} E_{1}(t)^{(1-\varepsilon) / 2} E_{2}(t)^{\varepsilon / 4}
$$

with $0<\varepsilon<1$, for $n=4$.

If $n=4$, let $V$ satisfy in addition (5.15) and

$$
\varphi_{2}(\rho) \leqq b_{2}\left(1+\rho^{p}\right)
$$

for some $p, 0 \leqq p<\infty$. Then in both cases, an a priori estimate of $E_{1}(t)$ implies an a priori estimate of $E_{2}(t)$.

Proof. The proof of part (1) is analogous to that of part (1) of Lemma 5.2 and will be omitted. In order to prove parts (2) and (3), we estimate the right hand side of (5.27). All the terms of order $e$ are estimated in the same way and we consider only $\left\langle D^{2} F,[D F, F]\right\rangle$. By Hölder's inequality, we obtain

$$
\int d x \mid\left\langle D^{2} F,[D F, F]\right\rangle \leqq C\left\|D^{2} F\right\|_{2}\|D F\|_{r}\|F\|_{q}
$$

with $1 / q+1 / r=1 / 2$. For $n=2$, we take $1 / q=\varepsilon / 2$ for some $\varepsilon, 0<\varepsilon<1$, and for $n=3,4$, we take $r=n$. From the covariant Sobolev inequalities we obtain for $n=2$

$$
\cdots \leqq C\left\|D^{2} F\right\|_{2}^{1+\varepsilon}\|D F\|_{2}^{2-2 \varepsilon}\|F\|_{2}^{\varepsilon} \leqq C E_{2}^{(1+\varepsilon) / 2} E_{1}^{1-\varepsilon} \bar{E}_{0}^{\varepsilon / 2},
$$

and for $n=3,4$,

$$
\cdots \leqq C\left\|D^{2} F\right\|_{2}^{n / 2}\|D F\|_{2}^{3-n / 2} \leqq C E_{2}^{n / 4} E_{1}^{3 / 2-n / 4} .
$$


We next estimate the term containing $V$ in the right hand side of (5.27). From the relation

$$
\begin{aligned}
D_{j} D_{\ell}\left(\phi V^{\prime}\right)= & V^{\prime} D_{j} \psi_{\ell}+2 V^{\prime \prime} \operatorname{Re}\left\langle\phi, D_{j} \psi_{\ell}\right\rangle \phi \\
& +V^{\prime \prime}\left\{2 \operatorname{Re}\left\langle\phi, \psi_{j}\right\rangle \psi_{\ell}+2 \operatorname{Re}\left\langle\phi, \psi_{\ell}\right\rangle \psi_{j}+2 \operatorname{Re}\left\langle\psi_{j}, \psi_{\ell}\right\rangle \phi\right\} \\
& +V^{\prime \prime \prime} 2 \operatorname{Re}\left\langle\phi, \psi_{j}\right\rangle 2 \operatorname{Re}\left\langle\phi, \psi_{\ell}\right\rangle \phi,
\end{aligned}
$$

it follows that

$$
\begin{aligned}
\int d x\left|\left\langle D^{j} D^{\ell} \psi_{0}, D_{j} D_{\ell}\left(\phi V^{\prime}\right)\right\rangle\right| \leqq & \left\|D^{2} \psi\right\|_{2} \\
& \times\left\||D \psi| \varphi_{1}\left(|\phi|^{2}\right)+|\psi|^{2} 2 \varphi_{2}\left(|\phi|^{2}\right)\right\|_{2} .
\end{aligned}
$$

For $n=2$ and 3, $\phi$ is estimated in $L^{\infty}$ through (5.14). For $n=4$, repeated use of the covariant Sobolev inequalities together with the constraint (2.8) yields in a similar way

$$
\|\phi\|_{\infty} \leqq C_{\varepsilon}\|\psi\|_{2}^{\varepsilon / 2}\|D \psi\|_{2}^{1-\varepsilon}\left\|D^{2} \psi\right\|_{2}^{\varepsilon / 2}
$$

which implies (5.31). One can then estimate the right hand side of (5.37) for $2 \leqq n \leqq 4$ by

$$
\begin{aligned}
\ldots \leqq & \left\|D^{2} \psi\right\|_{2}\left\{\|D \psi\|_{2} \varphi_{1}\left(\|\phi\|_{\infty}^{2}\right)\right. \\
& \left.+C\|D \psi\|_{2}^{n / 2}\|\psi\|_{2}^{2-n / 2} \varphi_{2}\left(\|\phi\|_{\infty}^{2}\right)\right\} .
\end{aligned}
$$

Together with (5.34), (5.35) and their analogues for the terms of order $e$ containing $\psi$ in the right hand side of (5.27), (5.39) yields the estimates (5.29) and (5.30). The last statement of part (2) of Lemma 5.3 then follows from Lemma 5.2, part (3) and from the fact that the right hand side of (5.29) is at most linear in $E_{2}$. Similarly, the last statement of part (3) follows from the fact that, given $\bar{E}_{0}$ and $E_{1}$, the right hand side of (5.30) is at most linear in $E_{2}$ under the assumptions made. This last fact follows directly from (5.14) for $n=3$, and for $n=4$, from (5.15), (5.32) and (5.31), possibly after replacing $\varepsilon$ by $\varepsilon / p$ if $p \leqq 1$ (cf. the proof of Lemma 5.2, part (3)).

Q.E.D.

Remark 5.2. Together with Remark 5.1, part (3) of Lemma 5.3 shows that for $n=3,4$, an a priori estimate of $F$ in $L^{n}$ implies an a priori estimate of $E_{2}$ if $V$ satisfies (5.15) with $p \leqq(n-2)^{-1}$, and for $n=4$, if $V$ satisfies in addition (5.32) for some $p, 0 \leqq p<\infty$.

Remark 5.3. We have not considered $E_{2}$ for $n=1$, because in this case $E_{2}$ is not defined for general $u_{0} \in \mathscr{H}^{1}$. However for $u_{0} \in \mathscr{H}^{2}$, also $u(t) \in \mathscr{H}^{2}$ by Proposition 2.2, so that the quantity $E_{2}$ is well defined. It satisfies part (1) of Lemma 5.3 and an estimate similar to (5.29) and is therefore estimated in terms of the initial data.

Lemma 5.3 completes the estimates needed on the gauge invariant quantities $E_{\ell}$. In order to obtain a priori bounds on the solutions $u$ of $(2.11)$ in $\mathscr{H}^{k}$, we need additional estimates on the potential $A$.

Lemma 5.4. Let $1 \leqq n \leqq 4$, let $k=[n / 2+1]$, let $V \in \mathscr{C}^{k+2}\left(\mathbb{R}^{+}\right)$satisfy (5.4). Let $u_{0} \in \mathscr{H}^{k}$ satisfy the constraints (2.6) and (2.8). Let I be an interval of $\mathbb{R}$ containing the 
origin and let $u \in \mathscr{C}\left(I, \mathscr{H}^{k}\right)$ be a solution of (2.11). Then for any $t \in I, A$ satisfies the following estimate

$$
\begin{aligned}
\left|\partial_{0}\|\partial A(t)\|_{2}\right| \leqq & C\left\{E_{1}(t)^{1 / 2}+E_{1}(t)^{n / 8} \bar{E}_{0}(t)^{1 / 2-n / 8}\right. \\
& \left.\times\|\partial A(t)\|_{2}^{n / 4}\|A(t)\|_{2}^{1-n / 4}\right\} .
\end{aligned}
$$

In particular $A$ satisfies an a priori estimate in $H^{1}$ in terms of $E_{1}$ and of the initial data.

Proof. As in the proof of part (1) of Lemma 5.2, it is sufficient to prove the result for $u_{0} \in \mathscr{H}^{k+1}$. In this case the quantity

$$
m_{1}(t)=\frac{1}{2} \int d x\left\langle\partial^{\ell} A^{j}, \partial_{\ell} A_{j}\right\rangle
$$

is differentiable in time with derivative

$$
\partial_{0} m_{1}(t)=\int d x\left\{\left\langle\partial^{\ell} A^{j}, D^{\ell} F_{0 j}\right\rangle-e\left\langle\partial^{\ell} A^{j},\left[A_{\ell}, F_{0 j}\right]\right\rangle\right\} .
$$

Therefore, by Hölder's inequality

$$
\partial_{0}\|\partial A\|_{2} \leqq\|D F\|_{2}+C\|A\|_{4}\|F\|_{4},
$$

and by the covariant Sobolev inequality

$$
\partial_{0}\|\partial A\|_{2} \leqq\|D F\|_{2}+C\|D F\|_{2}^{n / 4}\|F\|_{2}^{1-n / 4}\|\partial A\|_{2}^{n / 4}\|A\|_{2}^{1-n / 4},
$$

from which (5.40) follows. The last statement follows from Lemma 5.1 and the fact that the right hand side of (5.40) is at most linear in $\|\partial A\|_{2}$.

Q.E.D.

Lemma 5.5. Let $2 \leqq n \leqq 4$, let $k=[n / 2+1]$, let $V \in \mathscr{C}^{k+2}\left(\mathbb{R}^{+}\right)$satisfy (5.4). Let $u_{0} \in \mathscr{H}^{k}$ satisfy the constraints (2.6) and (2.8). Let I be an interval of $\mathbb{R}$ containing the origin and let $u \in \mathscr{C}\left(I, \mathscr{H}^{k}\right)$ be a solution of (2.11). Then, for any $t \in I, A$ satisfies an a priori estimate in $H^{2}$ in terms of the initial data and of $E_{1}$ and $E_{2}$.

Proof. The proof is analogous to that of Lemma 5.4. We consider the quantity

$$
m_{2}(t)=-\frac{1}{2} \int d x\left\langle\partial^{k} \partial^{\ell} A^{j}, \partial_{k} \partial_{\ell} A_{j}\right\rangle
$$

with time derivative

$$
\partial_{0} m_{2}(t)=-\int d x\left\langle\partial^{k} \partial^{\ell} A^{j}, \partial_{k} \partial_{\ell} F_{0 j}\right\rangle
$$

so that by Schwarz's inequality

$$
\partial_{0}\left\|\partial^{2} A\right\|_{2} \leqq\left\|\partial^{2} F\right\|_{2} .
$$

One then expresses $\partial^{2} F$ in terms of covariant derivatives and obtains, in addition to $D^{2} F$, terms of several types, which are estimated as follows :

$$
\begin{aligned}
& \|A D F\|_{2} \leqq C\left\|D^{2} F\right\|_{2}^{n / 4}\|D F\|_{2}^{1-n / 4}\|\partial A\|_{2}^{n / 4}\|A\|_{2}^{1-n / 4}, \\
& \|(\partial A) F\|_{2} \leqq C\|D F\|_{2}^{n / 4}\|F\|_{2}^{1-n / 4}\left\|\partial^{2} A\right\|_{2}^{n / 4}\|\partial A\|_{2}^{1-n / 4},
\end{aligned}
$$

and

$$
\left\|A^{2} F\right\|_{2} \leqq C_{\varepsilon}\|D F\|_{2}^{1-\varepsilon}\|F\|_{2}^{\varepsilon}\|\partial A\|_{2}^{1+\varepsilon}\|A\|_{2}^{1-\varepsilon}
$$


for $n=2$,

$$
\begin{aligned}
& \left\|A^{2} F\right\|_{2} \leqq C\|D F\|_{2}\|\partial A\|_{2}^{2} \quad \text { for } n=3, \\
& \left\|A^{2} F\right\|_{2} \leqq C_{\varepsilon}\left\|D^{2} F\right\|_{2}^{1-\varepsilon}\|D F\|_{2}^{\varepsilon}\left\|\partial^{2} A\right\|_{2}^{\varepsilon}\|\partial A\|_{2}^{2-\varepsilon}
\end{aligned}
$$

for $n=4$, where $0<\varepsilon<1$.

The lemma follows from these estimates and from Lemmas 5.1 and 5.4

Q.E.D.

The last step in the a priori control of the solutions of (2.11) in $\mathscr{H}^{k}$ consists in estimating the $L^{2}$-norms of the ordinary derivatives of the various components of $u$ in terms of the $L^{2}$-norms of the covariant derivatives. In order to cover all cases of interest with a compact notation, in the following lemma, we denote by $h$ a function from $\mathbb{R}^{n}$ to $\mathscr{G}$ or $\mathscr{F}$ or any direct sum of such spaces, and by $H^{k}$ the Sobolev spaces of such vector valued functions.

Lemma 5.6. (1) Let $h \in H^{1}$ and $A \in L^{\infty}+L^{q}$, namely $A=A_{1}+A_{2}$ with $A_{1} \in L^{\infty}$ and $A_{2} \in L^{q}$, where $q=2$ for $n=1, q>2$ for $n=2$, and $q=n$ for $n \geqq 3$. Then $h$ is estimated in $\mathrm{H}^{1}$ in terms of the $\mathrm{L}^{2}$-norms of $h$ and $\mathrm{Dh}$. More precisely

$$
\begin{aligned}
\|\partial h\|_{2} \leqq & \|D h\|_{2}+e\left\|A_{1}\right\|_{\infty}\|h\|_{2} \\
& +C\left\|A_{2}\right\|_{q}\|D h\|_{2}^{n / q}\|h\|_{2}^{1-n / q} .
\end{aligned}
$$

(2) Let $h \in H^{2}, A=A_{1}+A_{2}$ with $A_{1} \in L^{\infty}, A_{2} \in L^{q^{\prime}}$, and $A=A_{1}^{\prime}+A_{2}^{\prime}$ with $\partial A_{1}^{\prime} \in L^{\infty}$ and $\partial A_{2}^{\prime} \in L^{q^{\prime} / 2}$, where $q^{\prime}=4$ for $n \leqq 3, q^{\prime}>4$ for $n=4$, and $q^{\prime}=n$ for $n \geqq 5$. Then $h$ is estimated in $H^{2}$ in terms of the $L^{2}$-norms of $h, D h$ and $D^{2} h$ (through a formula similar to (5.45), but more complicated, see (5.46) below).

Proof. (1) The estimate (5.45) follows immediately from the definition of the covariant derivative through the Hölder and covariant Sobolev inequalities.

(2) Similarly, from

$$
\partial^{2} h=D^{2} h-2 e A D h-e(\partial A) h+e^{2} A^{2} h,
$$

one finds, using the Hölder and covariant Sobolev inequalities,

$$
\begin{aligned}
\left\|\partial^{2} h\right\|_{2} \leqq & \left\|D^{2} h\right\|_{2}+C\left\{\left\|A_{1}\right\|_{\infty}\|D h\|_{2}\right. \\
& +\left(\left\|\partial A_{1}^{\prime}\right\|_{\infty}+\left\|A_{1}\right\|_{\infty}^{2}\right)\|h\|_{2}+\left\|A_{2}\right\|_{q^{\prime}}\left\|D^{2} h\right\|_{2}^{n / q^{\prime}}\|D h\|_{2}^{1-n / q^{\prime}} \\
& \left.+\left(\left\|\partial A_{2}^{\prime}\right\|_{q^{\prime} / 2}+\left\|A_{2}\right\|_{q^{\prime}}^{2}\right)\|h\|_{r}\right\},
\end{aligned}
$$

where $1 / r+2 / q^{\prime}=1 / 2$. The last norm $\|h\|_{r}$ is itself estimated in terms of the $L^{2}$ norms of $h, D h$ and $D^{2} h$ by further use of the covariant Sobolev inequalities.

Q.E.D.

We are now in a position to state the global existence results. We first consider the theory in global spaces as described in Sect. 2.

Proposition 5.1. Let $n=1,2$, let $k=[n / 2+1]$ and let $V \in \mathscr{C}^{k+2}\left(\mathbb{R}^{+}\right)$satisfy (5.4). If $n=2$, in addition let $V$ satisfy (5.15) for some $p, 0 \leqq p<\infty$. Let $u_{0} \in \mathscr{H}^{k}$ satisfy the constraints (2.6) and (2.8). Then the equation (2.11) has a unique solution $u \in$ 
$\mathscr{C}\left(\mathbb{R}, \mathscr{H}^{k}\right)$ and $u$ satisfies the constraints (2.6) and (2.8) for all times. If in addition $u_{0}$ satisfies the constraint (2.7), then $u$ satisfies (2.7) for all times.

Proof. Let $u$ be a solution of (2.11) in an interval $I$ containing the origin. Then it follows from Lemmas 5.1 to 5.6 that $u(t)$ is estimated a priori in $\mathscr{H}^{k}$ in terms of $u_{0}$, uniformly on bounded subintervals of $I$. More precisely, one first estimates the $L^{2}$-norms of $F, A, \psi$ and $\phi$ by Lemma 5.1, part (2), then the quantity $E_{1}$ by Lemma 5.2, part (2) for $n=1$ and part (3) for $n=2$ and, for $n=2$ only, the quantity $E_{2}$ by Lemma 5.3, part (2). From this, one derives an estimate of $A$ in $H^{1}$ by Lemma 5.4, and, for $n=2$, of $A$ in $H^{2}$ by Lemma 5.5. Using these estimates, one can convert the estimate of $E_{1}$ into an estimate of $F$ and $\psi$ in $H^{1}$ by Lemma 5.6, part (1), and, for $n=2$, one can convert the estimate of $E_{2}$ into an estimate of $F$ and $\psi$ in $H^{2}$, by Lemma 5.6, part (2). Finally one estimates $\phi$ in $H^{k}$ by using the constraint (2.8), the estimates on $\psi$ and Lemma 5.6 again. The existence of global solutions of (2.11) follows from the a priori estimate of $u$ in $\mathscr{H}^{k}$ thereby obtained and from Proposition 2.1 by standard arguments. The last statement of Proposition 5.1 is a partial repetition from Proposition 2.3.

Q.E.D.

In four dimensional space time (namely for $n=3$ ), our estimates are not sufficient to prove the existence of global solutions of (2.11). Nevertheless, they yield the following partial result, which strengthens Corollary 4.1. of [11].

Proposition 5.2. Let $n=3$ let $V \in \mathscr{C}^{4}\left(\mathbb{R}^{+}\right)$satisfy (5.4) and (5.15) for some $p$, $0 \leqq p \leqq 1$. Let $u_{0} \in \mathscr{H}^{2}$ satisfy the constraints (2.6) and (2.8). Then the equation (2.11) has a (unique) solution $u \in \mathscr{C}\left(I, \mathscr{H}^{2}\right)$ for some interval I containing the origin, and either $u$ can be continued for all times, or $F$ is unbounded in the $L^{3}$-norm over the maximal interval of existence.

Proof. The result follows by the same argument as in the proof of Proposition 5.1 from the estimates obtained in Lemma 5.1, part (2), in Lemma 5.2, part (4), in Remark 5.1, in Lemma 5.3, part (3), and in Lemmas 5.4 to 5.6.

Remark 5.4. In space time dimensions three and four, namely for $n=2$ and $n=3$, the assumptions on the potential $V$ in Propositions 5.1 and 5.2 as well as in Proposition 5.3 and Remark 5.6 below, consist of the condition $V \in \mathscr{C}^{4}\left(\mathbb{R}^{+}\right)$, the lower bound (5.4) and the upper bound (5.15) with $0 \leqq p<\infty$ for $n=2$ and $0 \leqq p \leqq 1$ for $n=3$. In particular, they allow for potentials of the form

$$
V\left(|\phi|^{2}\right)=-a^{2}|\phi|^{2}+|\phi|^{2(p+1)}
$$

with $p$ a positive integer or $3 \leqq p<\infty$ for $n=2$, and for the often considered potential

$$
V\left(|\phi|^{2}\right)=-a^{2}|\phi|^{2}+|\phi|^{4}
$$

for $n=3$. These potentials have non trivial minima.

Remark 5.5. It is clear, but we have not checked in detail, that Proposition 5.2 can be extended to five dimensional space time (namely $n=4$ ) with $\mathscr{H}^{2}$ replaced by $\mathscr{H}^{3}, L^{3}$ by $L^{4}$, with $p$ restricted to $0 \leqq p \leqq 1 / 2$ in (5.15) and $V$ satisfying in addition (5.32) for some $p, 0 \leqq p \leqq \infty$. A complete proof would require an estimate of $E_{3}$ and of $A$ in $\mathscr{H}^{3}$. 
We finally prove the existence of global solutions of (2.11) for the theory in local spaces described in Sect. 4. For potentials with non trivial minima such as those described in Remark 5.4, this will allow in particular for initial data and solutions for which $|\phi|$ tends to a non zero value minimizing $V$ at space like infinity.

Proposition 5.3. Let $n=1,2$, let $k=[n / 2+1]$ and let $V$ satisfy the assumptions of Proposition 5.1. Let $u_{0} \in \mathscr{H}_{\mathrm{loc}}^{k}$ satisfy the constraints (2.6) and (2.8). Then the equation $(2.11)$ has a unique solution $u \in \mathscr{C}\left(\mathbb{R}, \mathscr{H}_{\mathrm{loc}}^{k}\right)$ and $u$ satisfies the constraints (2.6)and(2.8)for all times. If in addition $u_{0}$ satisfies the constraint (2.7), then $u$ satisfies (2.7) for all times.

Proof. The result follows from Proposition 5.1 through a cut-off procedure, as in the proof of Theorem 1.2 of [9]. We first prove that for any open ball $\Omega=B(0, R)$, there exists a (unique) $\mathscr{H}_{1 \text { loc }}^{k}$-valued solution $u^{R}$ of (2.11) in the double cone $\Delta(R) \equiv$ $Q(\Omega, R) \cup Q(\Omega,-R)$ (see (4.1)), in the sense that the restriction of $u^{R}$ to $Q(\Omega, R)$ is an $\mathscr{H}_{\text {loc }}^{k}$-valued solution of $(2.11)$ in $Q(\Omega, R)$ and that a similar property, defined by an obvious symmetry, holds in $Q(\Omega,-R)$. For this purpose, we pick a function $g^{R} \in \mathscr{C}_{0}^{\infty}$ such that $0 \leqq g^{R} \leqq 1, g^{R}=1$ in $B(0, R)$ and $g^{R}=0$ in $C B(0, R+1)$. For $u_{0} \in \mathscr{H}_{\text {loc }}^{k}$, we define the cutoff initial data $u_{0}^{R}$ by

$$
A^{R}=g^{R} A, F_{0}^{R}=g^{R} F_{0}, \phi^{R}=g^{R} \phi, \psi_{0}^{R}=g^{R} \psi_{0}, \psi_{j}^{R}=\left(\partial_{j}+e A_{j}^{R}\right) \phi^{R},
$$

and

$$
F_{j k}^{R}=\partial_{j} A_{k}^{R}-\partial_{k} A_{j}^{R}+e\left[A_{j}^{R}, A_{k}^{R}\right] .
$$

Then $u_{0}^{R} \in \mathscr{H}^{k}$, with Supp $u_{0}^{R} \subset \overline{B(0, R+1)}$, and $u_{0}^{R}$ still satisfies the constraints (2.6) and (2.8). Therefore, by Proposition 5.1, there exists $\bar{u}^{R} \in \mathscr{C}\left(\mathbb{R}, \mathscr{H}^{k}\right)$ satisfying (2.11) with $u_{0}$ replaced by $u_{0}^{R}$, for all times, and satisfying the constraints (2.6) and (2.8). From the fact that $U$ is an hyperbolic group and $f$ a local interaction, it follows in addition that the restriction $u^{R}$ of $\bar{u}^{R}$ to the double cone $\Delta(R)$ is an $\mathscr{H}_{\text {loc }}^{k}$-valued solution of $(2.11)$ in $\Delta(R)$.

In order to complete the proof, we pick an increasing sequence $\left\{R_{j}\right\}, j=1$, $2, \ldots$ tending of infinity, so that $\bigcup_{j} \Delta\left(R_{j}\right)=\mathbb{R}^{n+1}$. With each $R_{j}$ is associated an $\mathscr{H}_{\text {loc }}^{k}$-valued solution $u^{R_{j}}$ of $(2.11)^{j}$ in $\Delta\left(R_{j}\right)$, and by Proposition 3.1 , for $j \leqq \ell, u^{R_{j}}$ coincides with the restriction of $u^{R \ell}$ to $\Delta\left(R_{j}\right)$. We then define $u$ in $\mathbb{R}^{n+1}$ by the consistent set of conditions that for all $j=1,2, \ldots$, the restriction of $u$ to $\Delta\left(R_{j}\right)$ coincides with $u^{R_{j}}$. It is then straightforward to check that $u$ is an $\mathscr{H}_{\text {loc }}^{k}$-valued solution of (2.11) in $\mathbb{R}^{n+1}$ and satisfies (2.6) and (2.8) for all times.

Uniqueness follows from Proposition 3.1, while the last statement of Proposition 5.3 is a partial repetition from Proposition 4.3.

Q.E.D.

Remark 5.6. In four dimensional space time, Proposition 5.2 also extends to the theory in local spaces, with $\mathscr{H}^{2}$ replaced by $\mathscr{H}_{\text {loc }}^{2}$ and $L^{3}$ by $L_{\text {loc }}^{3}$. Similarly, Remark 5.4 should extend to the theory in local spaces with appropriate changes.

\section{Appendix}

In this Appendix we derive the Sobolev inequalities that are used in Sect. 5. Let $\mathscr{V}$ be a finite dimensional complex vector space with scalar product $\langle\cdot, \cdot\rangle$ and 
norm $|\cdot|=\langle\cdot, \cdot\rangle^{1 / 2}, u$ a function from $\mathbb{R}^{n}$ to $\mathscr{V}$, and $A_{j}, j=1, \ldots, n, n$ functions from $\mathbb{R}^{n}$ to the antihermitian matrices in $\mathscr{V}$. The covariant derivative is defined as $D_{j} u=\partial_{j} u+A_{j} u$. We shall denote by $\|\cdot\|_{q}$ the norm in $L^{q} \equiv L^{q}\left(\mathbb{R}^{n}\right)$ and we shall also use the notation $L^{q}$ for vector valued functions. The formulation of the following proposition is essentially taken from Nirenberg [8].

Proposition A.1. Let $n \geqq 1,1 \leqq q \leqq \infty, 1 \leqq r \leqq \infty$, and let $\sigma$ and $p$ satisfy $0 \leqq \sigma \leqq 1$, $1 \leqq p \leqq \infty$, and

$$
1 / p=(1-\sigma) / r+\sigma(1 / q-1 / n)
$$

Assume in addition that if $p=\infty$, then $r<\infty$ and $\sigma<1$. Let $A_{j}, j=1, \ldots, n$ be $n$ functions from $\mathbb{R}^{n}$ to the antihermitian matrices in $\mathscr{V}$. Then, there exists a constant $C$, depending only on $n, p, q, r$, such that, for any $u \in L^{r}$ for which $\partial_{j} u \in L^{q}$ and $A_{j} u \in L^{q}, j=$ $1, \ldots, n$, the following inequality holds:

$$
\||u|\|_{p} \leqq C\||u|\|_{r}^{1-\sigma}\left\{\sum_{1 \leqq j \leqq n}\left\|\left|D_{j} u\right|\right\|_{q}\right\}^{\sigma},
$$

provided in the case $r=\infty$ and $q<n$, we assume in addition that either $u$ tends to zero at infinity or $u \in L^{r_{0}}$ for some finite $r_{0}>0$.

Proof. Let $u$ be as in the assumptions of the proposition. For any $\varepsilon>0$ we define the real valued function $v$ by

$$
v(x)=\left(|u(x)|^{2}+\varepsilon^{2}\right)^{1 / 2}-\varepsilon .
$$

First we show that the distributional derivatives of $v$ are given by

$$
\partial_{j} v=\left(|u|^{2}+\varepsilon^{2}\right)^{-1 / 2} \operatorname{Re}\left\langle u, \partial_{j} u\right\rangle .
$$

This is obtained by a regularization technique. Let $\left\{u_{k}\right\}, k \geqq 1$, be a sequence of $\mathscr{C}^{\infty}$ functions with values in $\mathscr{V}$ such that $u_{k} \rightarrow u$ in $L_{\text {loc }}^{1}$ and almost everywhere, and $\partial_{j} u_{k} \rightarrow \partial_{j} u$ in $L_{\mathrm{loc}}^{1}, j=1, \ldots, n$. The corresponding $v_{k}$, defined by

$$
v_{k}(x)=\left(\left|u_{k}(x)\right|^{2}+\varepsilon^{2}\right)^{1 / 2}-\varepsilon,
$$

satisfy in an obvious way

$$
\partial_{j} v_{k}=\left(\left|u_{k}\right|^{2}+\varepsilon^{2}\right)^{-1 / 2} \operatorname{Re}\left\langle u_{k}, \partial_{j} u_{k}\right\rangle .
$$

From $\left|v_{k}-v\right| \leqq\left|u_{k}-u\right|$, it follows that $v_{k} \rightarrow v$ in $L_{\mathrm{loc}}^{1}$ so that $\partial_{j} v_{k} \rightarrow \partial_{j} v$ in $\mathscr{D}^{\prime}$. On the other hand, by using the fact that $u_{k} \rightarrow u$ almost everywhere and that $\partial_{j} u_{k} \rightarrow \partial_{j} u$ in $L_{\mathrm{loc}}^{1}$, one sees that the right hand side of (A.6) converges to the right hand side of (A.4) in $L_{1 \mathrm{loc}}^{1}$. This proves (A.4). Now since

$$
\operatorname{Re}\left\langle u, \partial_{j} u\right\rangle=\operatorname{Re}\left\langle u, D_{j} u\right\rangle,
$$

(A.4) implies

$$
\left|\partial_{j} v\right| \leqq\left|D_{j} u\right|
$$

so that, by applying the usual Sobolev inequalities [8] to the scalar function $v$, 
one obtains

$$
\begin{aligned}
\|v\|_{p} & \leqq C\|v\|_{r}^{1-\sigma}\left\{\sum_{1 \leqq j \leqq n}\left\|\partial_{j} v\right\|_{q}\right\}^{\sigma} \\
& \leqq C\|v\|_{r}^{1-\sigma}\left\{\sum_{1 \leqq j \leqq n}\left\|\mid D_{j} u\right\|_{q}\right\}^{\sigma} .
\end{aligned}
$$

The inequality (A.2) follows by taking the limit $\varepsilon \rightarrow 0$ in (A.8).

\section{Q.E.D.}

Acknowledgements. One of us (G. V.) is grateful to K. Chadan for the kind hospitality at the Laboratoire de Physique Théorique in Orsay, where part of this work was done.

\section{References}

1. Adams, R. A. : Sobolev spaces, New York: Academic Press, 1975

2. Christodoulou, D. : J. Math. Pure Appl. 60, 99-130 (1981)

3. Ginibre, J., Velo, G. : Phys. Lett. B, 99, 405-410 (1981)

4. Glassey, R. T., Strauss, W. A. : Commun. Math. Phys. 65, 1-13 (1979)

5. Glassey, R. T., Strauss, W. A. : Commun. Math. Phys. 67, 51-67 (1979)

6. Glassey, R. T., Strauss, W. A. : Some global solutions of the Yang Mills equations in Minkowski space, Commun. Math. Phys. 81,171-187 (1981)

7. Moncrief, V.: J. Math. Phys. 21, 2291-2296 (1980).

8. Nirenberg, L. : Ann Scuol. Norm. Sup. Pisa 13, 116-162 (1959)

9. Parenti, C., Strocchi, F., Velo, G. : Ann. Scuol. Norm. Sup. Pisa, Serie IV, 3, 443-500 (1976)

10. Segal, I. E.: Ann. Math. 78, 339-364 (1963)

11. Segal, I. E.: J. Funct. Anal. 33, 175-194 (1979)

12. Eardley, D., Moncrief, V. : The global existence of Yang-Mills-Higgs field in 4 dimensional Minkowski space. I. Local existence and smoothness properties, II. Completion of the proof. Commun. Math. Phys. (to appear).

Communicated by A. Jaffe

Received April 6, 1981 